\title{
The hydrochloride and hydrobromide salt forms of $(S)$-amphetamine
}

\author{
Lynn Dennany, Alan R. Kennedy* and Brandon Walker
}

Westchem, Department of Pure \& Applied Chemistry, University of Strathclyde, 295 Cathedral Street, Glasgow G1 1XL, Scotland Correspondence email: a.r.kennedy@strath.ac.uk

\begin{abstract}
(S)-Amphetamine hydrochloride, $\mathrm{C}_{9} \mathrm{H}_{14} \mathrm{ClN}$, has a $Z^{\prime}=6$ structure with six independent cation, anion pairs. That these are indeed crystallographically independent is supported by different packing orientations of the cations and by observation of a wide range of cation conformations generated by rotation about the phenyl- $\mathrm{CH}_{2}$ bond. The supramolecular contacts about the anions also differ such that both a wide variation in the geometry of the three $\mathrm{N}-\mathrm{H} \cdots \mathrm{Cl}$ hydrogen bonds formed by each chloride anion and differences in $\mathrm{C}-\mathrm{H} \cdots \mathrm{Cl}$ contacts are apparent. ( $S$ )-Amphetamine hydrobromide, $\mathrm{C}_{9} \mathrm{H}_{14} \mathrm{BrN}$, is broadly similar to the chloride in terms of cation conformation, the existance of three $\mathrm{N}-\mathrm{H} \cdots X$ hydrogen bond contacts per anion and the overall 2 dimensional hydrogen bonded sheet motif. However, only the chloride structure features organic bilayers and $Z^{\prime}>1$.
\end{abstract}

\section{Comment}

Salt selection or salt screening is a common technique used in the pharmacuetical industry to improve the physicochemical properties of potential Active Pharmaceutical Ingredients (APIs, Stahl \& Wermuth 2008). Studies on systematic series of crystal structures of phenylethylamine salts have been instigated with a view to determining relationships between crystal structure and the physicochemical properties of APIs (see for example Kennedy et al., 2011; Black et al., 2007; Briggs et al., 2012; Cruickshank et al., 2013). The ultimate goal of such studies is to understand and improve the pharmaceutical salt selection process. A member of the phenylethylamine class of molecules, amphetamine is popularly known as a stimulant and as a drug of abuse (Kilminster et al., 1977), but it also has legitimate pharmaceutical roles for instance in the treatment of attention defecit hyperactivity disorder and of narcolepsy (Wood et al., 2014). Despite the high profile of amphetamine, there have been relatively few structural studies of its salt forms. The sulfate has been crystallographically characterised and has been shown to undergo a temperature dependent phase transition (Pogorzelec-Glaser et al., 2009) and additionally the crystal structures of a dihydrogen phosphate salt and a salt formed with a pyrazol derivative are known (Hebert, 1978; Reviriego et al., 2006). The lack of any halide salt forms is surprising as the typical synthetic route for amphetamine initially produces the chloride salt. Additionally, the chloride salt is sometimes found in general circulaion, although the sulfate salt is that most commonly seized by law enforcement agencies (United Nations, 2006). Structures are known for the chloride salts of structurally related drugs of abuse such as methylamphetamine (Hakey et al., 2008) and the more complex, ring substituted species methylenedioxymethamphetamine (MDMA or ecstacy) and ethylone (Morimoto et al., 1998 ; Cameron et al., 2015).

Reaction of (S)-amphetamine free base with aqueous $\mathrm{HCl}$ or $\mathrm{HBr}$ gave (S)-amphetamine hydrochloride (I) and (S)amphetamine hydrobromide (II), respectively. (I) is unusual in that it crystallises with six cations and six anions per unit cell in the space group P $1\left(Z=Z^{\prime}=6\right)$, Figure 1 . Structures with $Z^{\prime}>1$ have drawn much attention for their ability to shed light on fundamental aspects of crystal theory (Steed \& Steed, 2015; Bernstein et al., 2008). As discussed in the review by Steed and Steed (2015), (I) is in some ways a typical example of the type of species that gives Z'>1 structures, being as it is a relatively small and enantiopure organic compound that crystallises in space group P1. Note that the diffraction data for (I) were non-merohedrally twinned by a $180^{\circ}$ rotation about 001 . This could be worrying as the presence of unidentified twinning can lead to false identification of structures with $Z^{\prime}>1$ (see for example Herbstein, 1964). 
However, inspection of Figure 2 shows that a $Z^{\prime}=1$ structure is not correct here. The cations in the layers parallel to the $a c$ plane form two independent rows parallel to the $c$ direction. In each row every third cation has a different orientation from the others, being rotated by approximately $180^{\circ}$ along its phenyl to $\mathrm{NH}_{3}$ axis.

The six independent cations of (I) also show distinct conformational variation. This is most easily seen with the $\mathrm{C} 2 \mathrm{C} 3 \mathrm{C} 4 \mathrm{C} 5$ and $\mathrm{C} 2 \mathrm{C} 3 \mathrm{C} 4 \mathrm{C} 9$ torsion angles (and their equivalents in other ions) which have ranges of -61.9 (5) to -69.3 (4) and 106.9 (4) to 115.4 (4) ${ }^{\circ}$ for five of the six cations, see Table 2 . The geometry of the sixth cation, that containing atom N5, lies significantly outside this range with equivalent angles of -52.7 (4) and 127.5 (3) ${ }^{\circ}$. The cations all have anti conformations with NCCC torsion angles that lie within $3.5^{\circ}$ of $180^{\circ}$. The previously described crystal structures of salt forms of amphetamine all have similar anti conformations (Herbert, 1978; Pogorzelec-Glaser et al., 2009; Reviriego et al., 2006) as does solution phase amphetamine (Neville et al., 1971). The structure of the bromide salt (II) is shown in Figure 3. Here the torsion angles $\mathrm{C} 2 \mathrm{C} 3 \mathrm{C} 4 \mathrm{C} 5$ and $\mathrm{C} 2 \mathrm{C} 3 \mathrm{C} 4 \mathrm{C} 9$ are -75.2 (2) and 104.5 (2) ${ }^{\circ}$. These values lie out-with the range seen for (I). The angle $\mathrm{N} 1 \mathrm{C} 2 \mathrm{C} 3 \mathrm{C} 4$ is 158.84 (17) ${ }^{\circ}$ for (II) and this too shows a modest conformational change from the range found for the cations of (I). These small differences do not however amount to evidence of two or more dramatically distinct conformer geometries as has been previously described for salt forms of related phenylethylamine species such as methylephedrine, psuedo-ephedrine and tyramine (Kennedy et al., 2011; Black et al., 2007; Briggs et al., 2012).

All six crystallographically independent $\mathrm{NH}_{3}$ groups in structure (I) utilise all three $\mathrm{H}$ atoms as single hydrogen bond donors. Both the $\mathrm{H}_{2} \mathrm{PO}_{4}$ salt and the room temperature phase of the $\mathrm{SO}_{4}$ salt show similar hydrogen-bonding behaviour by the amphetamine cation (Pogorzelec-Glaser et al., 2009; Hebert, 1978). Each $\mathrm{NH}_{3}$ group in (I) thus bonds to three chloride anions, two of which are related to each other by translational symmetry with the third being independent, see Table 3. Although each anion and each cation is involved in three hydrogen bonding interactions, there are subtle differences in geometry. These are best shown by comparing the environment of $\mathrm{Cl1}$ with that of $\mathrm{Cl}$. The $\mathrm{N}-\mathrm{H} \cdots \mathrm{Cl}$ angles of $\mathrm{Cl} 5$ are all near linear $\left(169.4\right.$ to $\left.171.3^{\circ}\right)$ whilst the three angles about Cl1 are 145.0, 158.9 and $167.9^{\circ}$. There are further small differences, for instance $\mathrm{Cl} 2$ makes a much shorter contact with a phenyl ring than any other chloride anion (C19$\mathrm{H} 19 \cdots \mathrm{Cl} 22.72$ and 3.562 (5) $\AA$ for $\mathrm{H} \cdots \mathrm{Cl}$ and $\mathrm{C} \cdots \mathrm{Cl}$ distances respectively). $\mathrm{Cl} 5$ has a similar but longer interaction whilst the other four anions do not make any such contact. The $\mathrm{N}-\mathrm{H} \cdots \mathrm{Cl}$ hydrogen bonds link all six fragments parallel to the crystallographic $a$ and $c$ directions. Both cations and anions act as three-connected nodes and so each 2 dimensional hydrogen bonded sheet can be described as a net with $(6,3)$ topology. The packing structure can be seen in Figure 2, note the organic bilayers and the hydrophobic and hydrophilic layers that alternate parallel to the $b$ axis.

Despite the larger size of bromide as compared to chloride, each bromide anion in (II) also makes three hydrogen bonds to three amphetamine cations, Table 4 . As the cations also act as three-connected nodes this structure, like that of (I), features a net with $(6,3)$ topology. In this case the 2 dimensional hydrogen bonded sheets are parallel to the $a b$ plane. In contrast to (I) there are no $\mathrm{C}-\mathrm{H} \cdots \mathrm{X}$ contacts of less than the sum of van der Waals radii in (II). Figure 4 shows the resulting packing. Like (I) there are alternating hydrophobic and hydrophilic layers but the structure of (II) lacks the organic bilayers found in (I).

There are now structures available for five mineral acid derived salt forms of (S)-amphetamine, the chloride, bromide and dihydrogen phosphate forms together with the high and low temperature sulfate forms. All are layer structures with two dimensional hydrogen bonded hydrophilic layers alternating with organic, hydrophobic layers. A MERCURY packing analysis of the cation positions of the five structures suggests that on this basis only the two sulfate structures are related, having 18 matching cation positions from a cluster of 20 cations (RMS 0.791, Macrae et al., 2008). The difference between the two sulfate structures is as follows. Like (I), both sulfate structures consist of organic bilayers. However, the low temperature sulfate structure has two structurally distinct organic bilayers constructed from four crystallographically 
independent amphetamine cations (see Figure 5) whereas in the high temperature form all cations and hence all the organic layers are identical. The MERCURY packing analysis is thus highlighting the close match between the high temperature form's cation packing and the cation packing in only one of the two organic bilayers of the low temperature form. The packing in the second bilayer is different. Whilst (I) and the sulfate salt structures all feature organic bilayers, structure (II) and the $\mathrm{H}_{2} \mathrm{PO}_{4}$ salt structure do not. It is interesting that of the three bilayer structures, two have $\mathrm{Z}^{\prime}>1$ and the third is disordered. Both the other structures have $Z^{\prime}=1$ and are well ordered. Within these five structures, the formation of organic bilayers is correlated with an inability to form simple $Z^{\prime}=1$ ordered structures.

Although in all five salt structures each hydrophilic layer is interconnected by hydrogen bonds between cations and anions (and for the $\mathrm{H}_{2} \mathrm{PO}_{4}$ salt between anions and anions too), there are few strong or close contacts within the organic layers. There are some weak $\mathrm{C}-\mathrm{H} \cdots \pi$ contacts which seem to be influential in the transformation between the two sulfate phases (see Pogorzelec-Glaser et al., 2009 for a discussion). All structures form cation stacks within the organic layers but the constituent cations in each case are too far apart to form a bonded supramolecular motif. The exception is the bromide salt, (II). Here a close $\pi \cdots \pi$ contact does exist (shortest $\mathrm{C} \cdots \mathrm{C}$ distance 3.388 (3) $\AA$ for $\mathrm{C} 6$ to $\mathrm{C} 9^{\prime},{ }^{\prime}=\mathrm{x}+1, \mathrm{y}, \mathrm{z}$ ) and this connects stacks of cations parallel to the crystallographic $a$ direction.

\section{Synthesis and crystallization}

(S)-Amphetamine sulfate $(0.25 \mathrm{~g})$ was dissolved in $3 \mathrm{~cm}^{3}$ of deionized water. The $\mathrm{pH}$ of the solution was raised to approximately 12.5 by addition of sodium hydroxide solution and the organic product extracted into $5 \mathrm{~cm}^{3}$ of diethyl ether. Allowing the ether to evaporate yielded amphetamine free base as an oily residue. This oil was mixed with $2 \mathrm{~cm}^{2}$ of water and a few drops of either concentrated $\mathrm{HCl}$ or $\mathrm{HBr}$ were added and the resulting solution was warmed slightly. After several days of slow evaporation, colourless crystals of (I) and (II) had developed.

\section{Refinement}

Crystallographic measurements for (I) and (II) were carried out by the National Crystallography Service (Coles \& Gale, 2012). The data for (I) was treated as a non-merohedral twin by $180^{\circ}$ rotation about 001 . Applying the twin matrix -100 0 - 10.682001 within the CrystalClear software gave a reflection file in the SHELX hklf 5 format. The BASF parameter refined to 0.4431 (6). For both structures, $\mathrm{H}$ atoms bound to $\mathrm{C}$ atoms were placed in the expected geometric positions and treated in riding modes with $\mathrm{C}-\mathrm{H}=0.95,0.98,0.99$ and $1.00 \AA$ for $s p^{2} \mathrm{CH}$, methyl, $\mathrm{CH}_{2}$ and $s p^{3} \mathrm{CH}$ respectively. $\mathrm{U}(\mathrm{H})_{\text {iso }}$ $=1.5 \mathrm{U}(\mathrm{C})_{\mathrm{eq}}$ for methyl groups and $1.2 \mathrm{U}(\mathrm{C})_{\mathrm{eq}}$ for other $\mathrm{CH}$ groups. In (I) The $\mathrm{H}$ atoms of the $\mathrm{NH}_{3}$ groups were similarly modeled with $\mathrm{N}-\mathrm{H}=0.91 \AA$ and $\mathrm{U}(\mathrm{H})_{\text {iso }}=1.5 \mathrm{U}(\mathrm{N})_{\text {eq. }}$. However, for (II) the $\mathrm{H}$ atoms bound to $\mathrm{N}$ were refined isotropically, see Tables 3 and 4.

\section{Table 1}

Experimental details

\begin{tabular}{lll}
\hline & $(\mathrm{I})$ & $(\mathrm{II})$ \\
\hline Crystal data & & \\
Chemical formula & $\mathrm{C}_{9} \mathrm{H}_{14} \mathrm{~N} \cdot \mathrm{Cl}$ & $\mathrm{C}_{9} \mathrm{H}_{14} \mathrm{~N} \cdot \mathrm{Br}$ \\
$M_{\mathrm{r}}$ & 171.66 & 216.12 \\
Crystal system, space group & Triclinic, $P 1$ & Monoclinic, $P 2_{1}$ \\
Temperature $(\mathrm{K})$ & 100 & 100 \\
$a, b, c(\AA)$ & $5.6396(4), 16.3917(11), 16.9602(12)$ & $5.2366(4), 8.4264(5), 11.3895(8)$ \\
$\alpha, \beta, \gamma\left({ }^{\circ}\right)$ & $69.427(4), 89.995(5), 87.286(5)$ & $90,94.079(2), 90$ \\
$V\left(\AA^{3}\right)$ & $1465.98(18)$ & $501.30(6)$ \\
$Z$ & 6 & 2
\end{tabular}




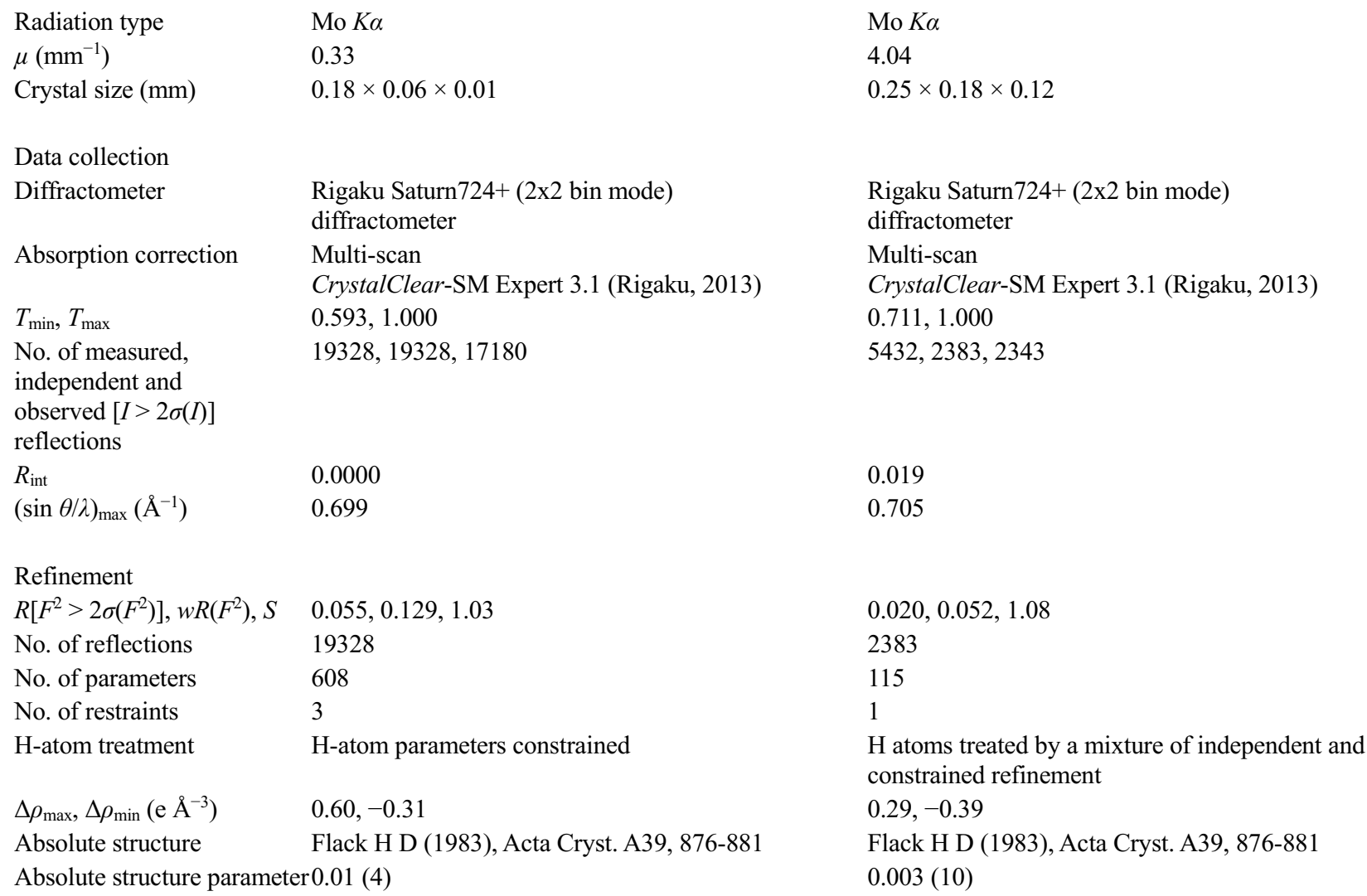

Computer programs: CrystalClear-SM Expert 3.1 b27 (Rigaku, 2012), SIR92 (Altomare et al., 1994, SIR97 (Altomare et al., 1994, SHELXL97 (Sheldrick, 2008), ORTEP-3 for Windows (Farrugia, 2012) \& Mercury (Macrae et al., 2008), SHELXL97.

\section{Table 2}

Selected torsion angles $\left({ }^{\circ}\right)$ for $(\mathrm{I})$

\begin{tabular}{llll}
\hline $\mathrm{N} 1-\mathrm{C} 2-\mathrm{C} 3-\mathrm{C} 4$ & $-177.0(3)$ & $\mathrm{N} 4-\mathrm{C} 32-\mathrm{C} 33-\mathrm{C} 34$ & $-179.0(3)$ \\
$\mathrm{C} 2-\mathrm{C} 3-\mathrm{C} 4-\mathrm{C} 5$ & $-69.3(4)$ & $\mathrm{C} 31-\mathrm{C} 32-\mathrm{C} 33-\mathrm{C} 34$ & $-57.9(4)$ \\
$\mathrm{C} 2-\mathrm{C} 3-\mathrm{C} 4-\mathrm{C} 9$ & $106.9(4)$ & $\mathrm{C} 32-\mathrm{C} 33-\mathrm{C} 34-\mathrm{C} 39$ & $109.7(4)$ \\
$\mathrm{N} 2-\mathrm{C} 12-\mathrm{C} 13-\mathrm{C} 14$ & $176.7(3)$ & $\mathrm{N} 5-\mathrm{C} 52-\mathrm{C} 53-\mathrm{C} 54$ & $179.3(3)$ \\
$\mathrm{C} 12-\mathrm{C} 13-\mathrm{C} 14-\mathrm{C} 15$ & $115.4(4)$ & $\mathrm{C} 52-\mathrm{C} 53-\mathrm{C} 54-\mathrm{C} 59$ & $-52.7(4)$ \\
$\mathrm{C} 12-\mathrm{C} 13-\mathrm{C} 14-\mathrm{C} 19$ & $-61.9(5)$ & $\mathrm{N} 6-\mathrm{C} 62-\mathrm{C} 63-\mathrm{C} 64$ & $127.5(3)$ \\
$\mathrm{N} 3-\mathrm{C} 22-\mathrm{C} 23-\mathrm{C} 24$ & $177.6(3)$ & $\mathrm{C} 62-\mathrm{C} 63-\mathrm{C} 64-\mathrm{C} 65$ & $178.6(3)$ \\
$\mathrm{C} 21-\mathrm{C} 22-\mathrm{C} 23-\mathrm{C} 24$ & $-61.3(4)$ & $\mathrm{C} 62-\mathrm{C} 63-\mathrm{C} 64-\mathrm{C} 69$ & $-63.9(4)$ \\
$\mathrm{C} 22-\mathrm{C} 23-\mathrm{C} 24-\mathrm{C} 29$ & $113.2(4)$ & & $114.7(4)$
\end{tabular}

\section{Table 3}

Hydrogen-bond geometry $\left(\AA,{ }^{\circ}\right)$ for (I)

\begin{tabular}{lllll}
\hline$D-\mathrm{H} \cdots A$ & $D-\mathrm{H}$ & $\mathrm{H} \cdots A$ & $D \cdots A$ & $D-\mathrm{H} \cdots A$ \\
\hline $\mathrm{N} 1-\mathrm{H} 1 A \cdots \mathrm{Cl} 5^{\mathrm{i}}$ & 0.91 & 2.40 & $3.304(3)$ & 171 \\
$\mathrm{~N} 1-\mathrm{H} 1 B \cdots \mathrm{Cl} 5^{\text {ii }}$ & 0.91 & 2.27 & $3.177(3)$ & 171 \\
$\mathrm{~N} 1-\mathrm{H} 1 C \cdots \mathrm{Cl} 6^{\mathrm{i}}$ & 0.91 & 2.28 & $3.181(3)$ & 169 \\
$\mathrm{~N} 2-\mathrm{H} 2 A \cdots \mathrm{Cl} 6^{\text {iii }}$ & 0.91 & 2.27 & $3.179(3)$ & 174 \\
$\mathrm{~N} 2-\mathrm{H} 2 B \cdots \mathrm{Cl} 1^{\text {iv }}$ & 0.91 & 2.43 & $3.215(3)$ & 145 \\
$\mathrm{~N} 2-\mathrm{H} 2 C \cdots \mathrm{Cl} 6^{\text {i }}$ & 0.91 & 2.37 & $3.233(3)$ & 158
\end{tabular}


research papers

\begin{tabular}{lllll}
$\mathrm{N} 3-\mathrm{H} 3 A \cdots \mathrm{Cl} 1^{\mathrm{iv}}$ & 0.91 & 2.28 & $3.151(3)$ & 159 \\
$\mathrm{~N} 3-\mathrm{H} 3 B \cdots \mathrm{Cl} 1$ & 0.91 & 2.22 & $3.118(3)$ & 168 \\
$\mathrm{~N} 3-\mathrm{H} 3 C \cdots \mathrm{Cl} 2$ & 0.91 & 2.27 & $3.171(3)$ & 169 \\
$\mathrm{~N} 4-\mathrm{H} 4 A \cdots \mathrm{Cl} 2$ & 0.91 & 2.25 & $3.149(3)$ & 168 \\
$\mathrm{~N} 4-\mathrm{H} 4 B \cdots \mathrm{Cl} 3$ & 0.91 & 2.26 & $3.164(3)$ & 172 \\
$\mathrm{~N} 4-\mathrm{H} 4 C \cdots \mathrm{Cl} 2^{\mathrm{v}}$ & 0.91 & 2.37 & $3.271(3)$ & 171 \\
$\mathrm{~N} 5-\mathrm{H} 5 A \cdots \mathrm{Cl} 3$ & 0.91 & 2.27 & $3.159(3)$ & 165 \\
$\mathrm{~N} 5-\mathrm{H} 5 B \cdots \mathrm{Cl} 4$ & 0.91 & 2.36 & $3.196(3)$ & 153 \\
$\mathrm{~N} 5-\mathrm{H} 5 C \cdots \mathrm{Cl} 3^{\mathrm{iv}}$ & 0.91 & 2.33 & $3.233(3)$ & 170 \\
$\mathrm{~N} 6-\mathrm{H} 6 A \cdots \mathrm{Cl} 4$ & 0.91 & 2.22 & $3.168(3)$ & 167 \\
$\mathrm{~N} 6-\mathrm{H} 6 B \cdots \mathrm{Cl} 5^{\mathrm{v}}$ & 0.91 & 2.27 & $3.158(3)$ & 169 \\
$\mathrm{~N} 6-\mathrm{H} 6 C \cdots \mathrm{Cl} 4^{\mathrm{v}}$ & 0.91 & 2.29 & & 161 \\
\hline
\end{tabular}

Symmetry codes: (i) $x, y, z+1$; (ii) $x-1, y, z+1$; (iii) $x+1, y, z+1$; (iv) $x+1, y, z$; (v) $x-1, y, z$.

\section{Table 4}

Hydrogen-bond geometry $\left(\AA,^{\circ}\right)$ for (II)

\begin{tabular}{lllll}
\hline$D-\mathrm{H} \cdots A$ & $D-\mathrm{H}$ & $\mathrm{H} \cdots A$ & $D \cdots A$ & $D-\mathrm{H} \cdots A$ \\
\hline $\mathrm{N} 1-\mathrm{H} 1 N \cdots \mathrm{Br} 1^{\mathrm{i}}$ & $0.83(3)$ & $2.54(3)$ & $3.3080(19)$ & $154(2)$ \\
$\mathrm{N} 1-\mathrm{H} 2 N \cdots \mathrm{Br} 1^{\mathrm{ii}}$ & $0.93(3)$ & $2.36(3)$ & $3.2656(19)$ & $165(3)$ \\
$\mathrm{N} 1-\mathrm{H} 3 N \cdots \mathrm{Br} 1$ & $0.94(3)$ & $2.44(3)$ & $3.3296(18)$ & $157(3)$ \\
\hline
\end{tabular}

Symmetry codes: (i) $-x+1, y-1 / 2,-z+1$; (ii) $x+1, y, z$.

\section{Acknowledgements}

We thank the National Crystallography Service (University of Southampton) for data collection on (I) and (II).

\section{References}

Altomare, A., Cascarano, G., Giacovazzo, C., Guagliardi, A., Burla, M. C., Polidori, G. \& Camalli, M. (1994). J. Appl. Cryst. 27, 435.

Bernstein, J., Dunitz, J. D. \& Gavezzotti, A. (2008). Cryst. Growth Des. 8, 2011-2018.

Black, S. N., Collier, E. A., Davey, R. J. \& Roberts, R. J. (2007). Pharm. Sci. 96, 1053-1068.

Briggs, N. E. B., Kennedy, A. R. \& Morrison, C. A. (2012). Acta Cryst. B68, 453-464.

Cameron, T. S., Grossert, J. S., Maheux, C. R., Alarcon, I. Q., Copeland, C. R. \& Linden, A. (2015). Acta Cryst. C71, 266-270.

Coles, S. J. \& Gale, P. A. (2012). Chem. Sci. 3, 683-689.

Cruickshank, L., Kennedy, A. R. \& Shankland, N. (2013). J. Mol. Struct. 1051, 132-136.

Farrugia, L. J. (2012). J. Appl. Cryst. 45, 849-854.

Hakey, P., Ouellette, W., Zubieta, J. \& Korter, T. (2008). Acta Cryst. E64, o940.

Hebert, H. (1978). Acta Cryst. B34, 611-615.

Herbstein, F. H. (1964). Acta Cryst. 17, 1094-1096.

Kennedy, A. R., Morrison, C. A., Briggs, N. E. B. \& Arbuckle, W. (2011). Cryst. Growth Des. 11, $1821-1834$.

Kilminster, I., Clarke, E. \& Taylor, P. (1977). WIK 2, Chiswick Publishing Ltd, London, England.

Macrae, C. F., Bruno, I. J., Chisholm, J. A., Edgington, P. R., McCabe, P., Pidcock, E., Rodriguez Monge, L., Taylor, R., van de Streek, J. \& Wood, P. A. (2008). J. Appl. Cryst. 41, 466-470. 
Morimoto, B. H., Lovell, S. \& Kahr, B. (1998). Acta Cryst. C54, 229-231.

Neville, G. A., Deslauriers, R., Blackburn, B. J. \& Smith, I. C. P. (1971). J. Med. Chem. 14, 717-721.

Pogorzelec-Glaser, K., Kaszynska, J., Rachocki, A., Tritt-Goc, J. \& Pietraszko, A. (2009). New J. Chem., 33, 1894-1900.

Reviriego, F., Rodriguez-Franco, M. I., Navarro, P., Garcia-Espana, E., Liu-Gonzalez, M., Verdejo, B. \& Domenech, A. (2006). J. Am. Chem. Soc., 128, 16458-16459.

Rigaku (2012). CrystalClear. Rigaku/MSC Inc., The Woodlands, Texas, USA.

Stahl, P. H. \& Wermuth, C. G. (2008). Eds. Handbook of Pharmaceutical Salts: Properties, Selection and Use. VHCA: Zurich.

Steed, K. M. \& Steed, J. W. (2015). Chem. Rev., 115, 2895-2933.

United Nations, Office of Drugs and Crime. (2006). Recommended methods for the identification and analysis of amphetamine, methamphetamine and their ring substituted analogues in seized materials. ST/NAR/34. UN: New York.

Wood, S., Sage, J. R., Shuman, T. \& Anagnostaras, S. G. (2014). Pharm. Rev., 66, 193-221.

\section{Figure 1}

Figure 1 . The molecular structure of (I) with non-H atoms shown as $50 \%$ probability ellipsoids.

\section{Figure 2}

Figure 2. Packing structure of (I) viewed down the crystallographic $a$ direction. The red and blue ellipsoids highlight the different cation orientations found in a cation row parallel to $c$. The neighbouring cation row of the same organic bilayer has a simlar 2 to 1 arrangement of cation orientations.

\section{Figure 3}

Figure 3. The molecular structure of (II) with non-H atoms shown as 50\% probability ellipsoids.

\section{Figure 4}

Figure 4. Packing structure of (II) viewed down the crystallographic $a$ direction. Note that the organic bilayers shown in Figure 3 are absent here.

\section{Figure 5}

Figure 5. Packing structure of the low temperature form of (s)-amphetamine sulfate drawn from AMPETS01. Note that the organic bilayers at $c=0$ and $c=1$ differ from the bilayer at $c=0.5$. 


\section{supplementary materials}

\section{The hydrochloride and hydrobromide salt forms of (S)-amphetamine}

\section{Computing details}

For both compounds, data collection: CrystalClear-SM Expert 3.1 b27 (Rigaku, 2012); cell refinement: CrystalClear-SM Expert 3.1 b27 (Rigaku, 2012); data reduction: CrystalClear-SM Expert 3.1 b27 (Rigaku, 2012). Program(s) used to solve structure: SIR92 (Altomare et al., 1994 for (I); SIR97 (Altomare et al., 1994 for (II). For both compounds, program(s) used to refine structure: SHELXL97 (Sheldrick, 2008); molecular graphics: ORTEP-3 for Windows (Farrugia, 2012) \& Mercury (Macrae et al., 2008); software used to prepare material for publication: SHELXL97.

(I)

\section{Crystal data}

$\mathrm{C}_{9} \mathrm{H}_{14} \mathrm{~N} \cdot \mathrm{Cl}$

$M_{r}=171.66$

Triclinic, $P 1$

Hall symbol: P 1

$a=5.6396$ (4) $\AA$

$b=16.3917(11) \AA$

$c=16.9602(12) \AA$

$\alpha=69.427(4)^{\circ}$

$\beta=89.995(5)^{\circ}$

$\gamma=87.286(5)^{\circ}$

$V=1465.98(18) \AA^{3}$

\section{Data collection}

Rigaku Saturn724+ (2x2 bin mode) diffractometer

Radiation source: fine-focus sealed tube Graphite monochromator

Detector resolution: 28.5714 pixels $\mathrm{mm}^{-1}$

profile data from $\omega$-scans

Absorption correction: multi-scan

CrystalClear-SM Expert 3.1 (Rigaku, 2013)

$T_{\min }=0.593, T_{\max }=1.000$

Refinement

Refinement on $F^{2}$

Least-squares matrix: full

$R\left[F^{2}>2 \sigma\left(F^{2}\right)\right]=0.055$

$w R\left(F^{2}\right)=0.129$

$S=1.03$

19328 reflections

608 parameters

3 restraints

Primary atom site location: structure-invariant direct methods

Secondary atom site location: difference Fourier map

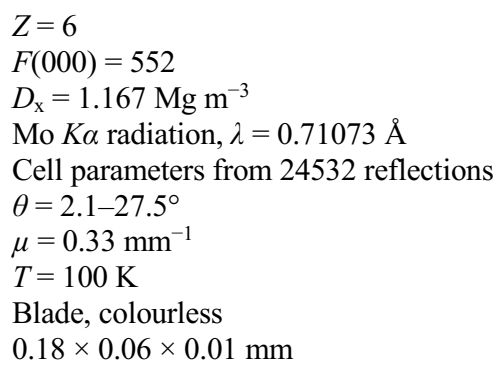

19328 measured reflections

19328 independent reflections

17180 reflections with $I>2 \sigma(I)$

$R_{\text {int }}=0.0000$

$\theta_{\max }=29.8^{\circ}, \theta_{\min }=1.5^{\circ}$

$h=-7 \rightarrow 7$

$k=-22 \rightarrow 22$

$l=-23 \rightarrow 22$

Hydrogen site location: inferred from neighbouring sites

$\mathrm{H}$-atom parameters constrained

$w=1 /\left[\sigma^{2}\left(F_{\mathrm{o}}^{2}\right)+(0.070 P)^{2}+0.9426 P\right]$

where $P=\left(F_{\mathrm{o}}{ }^{2}+2 F_{\mathrm{c}}{ }^{2}\right) / 3$

$(\Delta / \sigma)_{\max }=0.001$

$\Delta \rho_{\max }=0.60$ e $\AA^{-3}$

$\Delta \rho_{\min }=-0.31$ e $\AA^{-3}$

Absolute structure: Flack H D (1983), Acta Cryst.

A39, 876-881

Absolute structure parameter: 0.01 (4) 


\section{Special details}

Refinement

Refinement of $F^{2}$ against ALL reflections. The weighted $R$-factor $w R$ and goodness of fit $S$ are based on $F^{2}$, conventional $R$-factors $R$ are based on $F$, with $F$ set to zero for negative $F^{2}$. The threshold expression of $F^{2}>2 \sigma\left(F^{2}\right)$ is used only for calculating $R$-factors (gt) etc. and is not relevant to the choice of reflections for refinement. $R$-factors based on $F^{2}$ are statistically about twice as large as those based on $F$, and $R$ - factors based on ALL data will be even larger.

Fractional atomic coordinates and isotropic or equivalent isotropic displacement parameters $\left(\AA^{2}\right)$ for (I)

\begin{tabular}{|c|c|c|c|c|}
\hline & $x$ & $y$ & $z$ & $U_{\text {iso }} * / U_{\text {eq }}$ \\
\hline $\mathrm{Cl1}$ & $-0.06769(12)$ & $1.62848(5)$ & $0.75422(6)$ & $0.02168(17)$ \\
\hline $\mathrm{Cl} 2$ & $0.53180(13)$ & $1.80851(5)$ & $0.53529(6)$ & $0.02264(18)$ \\
\hline $\mathrm{Cl} 3$ & $0.06999(12)$ & $1.61130(5)$ & $0.42734(5)$ & 0.01902 (16) \\
\hline $\mathrm{Cl} 4$ & $0.41074(12)$ & $1.78429(5)$ & $0.20307(5)$ & $0.01911(16)$ \\
\hline $\mathrm{Cl} 5$ & $0.80481(13)$ & $1.59774(5)$ & $0.10755(5)$ & $0.02096(17)$ \\
\hline $\mathrm{Cl} 6$ & $0.27312(12)$ & $1.80318(5)$ & $-0.14216(5)$ & $0.02188(17)$ \\
\hline N1 & $0.2889(5)$ & $1.61990(18)$ & $1.00800(18)$ & $0.0206(6)$ \\
\hline H1A & 0.4237 & 1.6091 & 1.0403 & $0.031 *$ \\
\hline H1B & 0.1600 & 1.6125 & 1.0417 & $0.031 *$ \\
\hline $\mathrm{H} 1 \mathrm{C}$ & 0.2850 & 1.6757 & 0.9708 & $0.031 *$ \\
\hline $\mathrm{N} 2$ & $0.7768(5)$ & $1.81575(18)$ & $0.76431(19)$ & $0.0231(6)$ \\
\hline $\mathrm{H} 2 \mathrm{~A}$ & 0.9174 & 1.8162 & 0.7905 & $0.035^{*}$ \\
\hline $\mathrm{H} 2 \mathrm{~B}$ & 0.7879 & 1.7752 & 0.7390 & $0.035^{*}$ \\
\hline $\mathrm{H} 2 \mathrm{C}$ & 0.6596 & 1.8023 & 0.8030 & $0.035^{*}$ \\
\hline N3 & $0.4294(5)$ & $1.62596(18)$ & $0.67440(17)$ & $0.0176(6)$ \\
\hline $\mathrm{H} 3 \mathrm{~A}$ & 0.5599 & 1.6148 & 0.7088 & $0.026^{*}$ \\
\hline H3B & 0.2964 & 1.6246 & 0.7051 & $0.026^{*}$ \\
\hline $\mathrm{H} 3 \mathrm{C}$ & 0.4372 & 1.6796 & 0.6338 & $0.026^{*}$ \\
\hline N4 & $0.0460(5)$ & $1.79490(17)$ & $0.45030(18)$ & $0.0189(6)$ \\
\hline $\mathrm{H} 4 \mathrm{~A}$ & 0.1729 & 1.8016 & 0.4803 & $0.028^{*}$ \\
\hline $\mathrm{H} 4 \mathrm{~B}$ & 0.0523 & 1.7398 & 0.4491 & $0.028^{*}$ \\
\hline $\mathrm{H} 4 \mathrm{C}$ & -0.0906 & 1.8043 & 0.4751 & $0.028^{*}$ \\
\hline N5 & $0.5642(5)$ & $1.60012(17)$ & $0.34301(17)$ & $0.0172(6)$ \\
\hline $\mathrm{H} 5 \mathrm{~A}$ & 0.4378 & 1.6004 & 0.3763 & $0.026^{*}$ \\
\hline H5B & 0.5403 & 1.6431 & 0.2919 & $0.026^{*}$ \\
\hline $\mathrm{H} 5 \mathrm{C}$ & 0.6984 & 1.6095 & 0.3677 & $0.026^{*}$ \\
\hline N6 & $-0.0844(5)$ & $1.77880(17)$ & $0.12398(17)$ & $0.0182(6)$ \\
\hline H6A & 0.0471 & 1.7787 & 0.1551 & $0.027 *$ \\
\hline H6B & -0.0951 & 1.7253 & 0.1196 & $0.027 *$ \\
\hline $\mathrm{H} 6 \mathrm{C}$ & -0.2158 & 1.7913 & 0.1496 & $0.027 *$ \\
\hline $\mathrm{C} 1$ & $0.5080(7)$ & $1.5677(3)$ & $0.9095(3)$ & $0.0304(9)$ \\
\hline H1D & 0.5137 & 1.6283 & 0.8710 & $0.046^{*}$ \\
\hline H1E & 0.5085 & 1.5288 & 0.8770 & $0.046^{*}$ \\
\hline $\mathrm{H} 1 \mathrm{~F}$ & 0.6469 & 1.5529 & 0.9476 & $0.046^{*}$ \\
\hline $\mathrm{C} 2$ & $0.2852(6)$ & $1.5573(2)$ & $0.9602(2)$ & $0.0203(7)$ \\
\hline $\mathrm{H} 2$ & 0.1446 & 1.5733 & 0.9209 & $0.024 *$ \\
\hline $\mathrm{C} 3$ & $0.2596(6)$ & $1.4649(2)$ & $1.0243(2)$ & $0.0228(7)$ \\
\hline $\mathrm{H} 3 \mathrm{D}$ & 0.3895 & 1.4511 & 1.0670 & $0.027 *$ \\
\hline H3E & 0.1072 & 1.4628 & 1.0538 & $0.027^{*}$ \\
\hline $\mathrm{C} 4$ & $0.2671(6)$ & $1.3968(2)$ & $0.9828(2)$ & $0.0203(7)$ \\
\hline $\mathrm{C} 5$ & $0.0827(6)$ & $1.3923(2)$ & $0.9303(2)$ & $0.0218(7)$ \\
\hline H5 & -0.0537 & 1.4309 & 0.9220 & $0.026^{*}$ \\
\hline C6 & $0.0963(6)$ & $1.3318(2)$ & $0.8898(2)$ & $0.0233(8)$ \\
\hline H6 & -0.0311 & 1.3290 & 0.8543 & $0.028^{*}$ \\
\hline
\end{tabular}


supplementary materials

\begin{tabular}{|c|c|c|c|c|}
\hline $\mathrm{C7}$ & $0.2959(6)$ & $1.2751(2)$ & 0.9009 (3) & $0.0226(8)$ \\
\hline $\mathrm{H} 7$ & 0.3054 & 1.2338 & 0.8732 & $0.027^{*}$ \\
\hline $\mathrm{C} 8$ & $0.4794(7)$ & $1.2797(2)$ & $0.9526(2)$ & $0.0244(8)$ \\
\hline H8 & 0.6153 & 1.2410 & 0.9608 & $0.029 *$ \\
\hline C9 & $0.4681(6)$ & $1.3400(2)$ & $0.9928(2)$ & $0.0215(8)$ \\
\hline H9 & 0.5974 & 1.3430 & 1.0274 & $0.026^{*}$ \\
\hline C11 & $0.6831(8)$ & $1.9714(3)$ & $0.7410(3)$ & $0.0401(10)$ \\
\hline H11A & 0.8307 & 1.9747 & 0.7700 & $0.060^{*}$ \\
\hline H11B & 0.5562 & 1.9535 & 0.7821 & $0.060^{*}$ \\
\hline $\mathrm{H} 11 \mathrm{C}$ & 0.6392 & 2.0288 & 0.6990 & $0.060^{*}$ \\
\hline $\mathrm{C} 12$ & $0.7190(7)$ & $1.9059(3)$ & $0.6978(2)$ & $0.0289(8)$ \\
\hline H12 & 0.5661 & 1.9034 & 0.6691 & $0.035^{*}$ \\
\hline $\mathrm{C} 13$ & $0.9113(7)$ & $1.9288(3)$ & $0.6319(2)$ & 0.0299 (8) \\
\hline H13A & 0.9293 & 1.8826 & 0.6070 & $0.036^{*}$ \\
\hline H13B & 1.0646 & 1.9319 & 0.6589 & $0.036^{*}$ \\
\hline C14 & $0.8476(7)$ & $2.0172(2)$ & $0.5618(2)$ & $0.0292(8)$ \\
\hline $\mathrm{C} 15$ & $0.9846(8)$ & $2.0886(3)$ & $0.5489(3)$ & $0.0479(12)$ \\
\hline $\mathrm{H} 15$ & 1.1239 & 2.0830 & 0.5822 & $0.057^{*}$ \\
\hline $\mathrm{C} 16$ & $0.9201(9)$ & $2.1672(3)$ & $0.4881(3)$ & $0.0502(13)$ \\
\hline H16 & 1.0156 & 2.2156 & 0.4798 & $0.060 *$ \\
\hline $\mathrm{C} 17$ & $0.7193(7)$ & $2.1771(3)$ & $0.4391(2)$ & $0.0342(9)$ \\
\hline H17 & 0.6785 & 2.2315 & 0.3962 & $0.041^{*}$ \\
\hline $\mathrm{C} 18$ & $0.5783(7)$ & $2.1078(2)$ & $0.4525(3)$ & 0.0368 (9) \\
\hline H18 & 0.4352 & 2.1150 & 0.4207 & $0.044^{*}$ \\
\hline C19 & $0.6437(7)$ & $2.0277(2)$ & $0.5121(3)$ & $0.0356(9)$ \\
\hline H19 & 0.5489 & 1.9793 & 0.5194 & $0.043^{*}$ \\
\hline $\mathrm{C} 21$ & $0.6330(6)$ & $1.5667(2)$ & $0.5781(2)$ & $0.0235(8)$ \\
\hline $\mathrm{H} 21 \mathrm{~A}$ & 0.6239 & 1.6241 & 0.5331 & $0.035^{*}$ \\
\hline $\mathrm{H} 21 \mathrm{~B}$ & 0.6340 & 1.5210 & 0.5532 & $0.035^{*}$ \\
\hline $\mathrm{H} 21 \mathrm{C}$ & 0.7789 & 1.5604 & 0.6115 & $0.035^{*}$ \\
\hline $\mathrm{C} 22$ & $0.4206(6)$ & $1.5584(2)$ & $0.6341(2)$ & $0.0157(6)$ \\
\hline $\mathrm{H} 22$ & 0.2732 & 1.5702 & 0.5983 & $0.019^{*}$ \\
\hline $\mathrm{C} 23$ & $0.4086(6)$ & $1.4677(2)$ & $0.7034(2)$ & $0.0227(7)$ \\
\hline $\mathrm{H} 23 \mathrm{~A}$ & 0.5530 & 1.4557 & 0.7396 & $0.027 *$ \\
\hline $\mathrm{H} 23 \mathrm{~B}$ & 0.2697 & 1.4678 & 0.7390 & $0.027 *$ \\
\hline $\mathrm{C} 24$ & $0.3889(6)$ & $1.3964(2)$ & $0.6675(2)$ & $0.0192(7)$ \\
\hline $\mathrm{C} 25$ & $0.1894(6)$ & $1.3917(2)$ & $0.6201(3)$ & $0.0270(8)$ \\
\hline $\mathrm{H} 25$ & 0.0620 & 1.4341 & 0.6117 & $0.032 *$ \\
\hline $\mathrm{C} 26$ & $0.1718(6)$ & $1.3279(2)$ & $0.5856(2)$ & $0.0272(8)$ \\
\hline $\mathrm{H} 26$ & 0.0332 & 1.3263 & 0.5544 & $0.033^{*}$ \\
\hline $\mathrm{C} 27$ & $0.3570(6)$ & $1.2656(2)$ & $0.5963(2)$ & $0.0230(8)$ \\
\hline $\mathrm{H} 27$ & 0.3461 & 1.2215 & 0.5723 & $0.028^{*}$ \\
\hline $\mathrm{C} 28$ & $0.5574(6)$ & $1.2685(2)$ & $0.6422(2)$ & $0.0261(8)$ \\
\hline $\mathrm{H} 28$ & 0.6856 & 1.2266 & 0.6495 & $0.031 *$ \\
\hline $\mathrm{C} 29$ & $0.5706(6)$ & $1.3334(2)$ & $0.6780(2)$ & $0.0235(7)$ \\
\hline $\mathrm{H} 29$ & 0.7077 & 1.3342 & 0.7102 & $0.028^{*}$ \\
\hline $\mathrm{C} 31$ & $-0.1718(7)$ & $1.8495(2)$ & $0.3151(2)$ & $0.0286(9)$ \\
\hline H31A & -0.1736 & 1.7897 & 0.3151 & $0.043^{*}$ \\
\hline H31B & -0.1710 & 1.8905 & 0.2569 & $0.043^{*}$ \\
\hline $\mathrm{H} 31 \mathrm{C}$ & -0.3133 & 1.8620 & 0.3432 & $0.043^{*}$ \\
\hline C32 & $0.0519(6)$ & $1.8592(2)$ & $0.3622(2)$ & $0.0173(7)$ \\
\hline H32 & 0.1942 & 1.8440 & 0.3340 & $0.021 *$ \\
\hline C33 & $0.0743(6)$ & $1.9512(2)$ & $0.3631(2)$ & $0.0214(7)$ \\
\hline $\mathrm{H} 33 \mathrm{~A}$ & -0.0604 & 1.9657 & 0.3941 & $0.026^{*}$ \\
\hline
\end{tabular}


supplementary materials

\begin{tabular}{|c|c|c|c|c|}
\hline H33B & 0.2228 & 1.9533 & 0.3933 & $0.026^{*}$ \\
\hline C34 & $0.0766(6)$ & $2.0180(2)$ & $0.2751(2)$ & $0.0201(7)$ \\
\hline C35 & $0.2649(6)$ & 2.0187 (2) & $0.2217(2)$ & $0.0243(8)$ \\
\hline H35 & 0.3973 & 1.9786 & 0.2420 & $0.029 *$ \\
\hline C36 & $0.2624(7)$ & 2.0769 (2) & 0.1395 (3) & $0.0281(8)$ \\
\hline H36 & 0.3936 & 2.0767 & 0.1044 & $0.034 *$ \\
\hline C37 & $0.0690(6)$ & $2.1358(2)$ & 0.1077 (2) & $0.0244(8)$ \\
\hline H37 & 0.0669 & 2.1752 & 0.0511 & $0.029 *$ \\
\hline $\mathrm{C} 38$ & $-0.1205(7)$ & $2.1360(2)$ & $0.1599(2)$ & $0.0252(8)$ \\
\hline H38 & -0.2535 & 2.1757 & 0.1392 & $0.030 *$ \\
\hline C39 & $-0.1148(6)$ & $2.0778(2)$ & $0.2431(2)$ & $0.0214(7)$ \\
\hline H39 & -0.2443 & 2.0789 & 0.2786 & $0.026^{*}$ \\
\hline C51 & $0.6355(6)$ & 1.4427 (2) & $0.4188(2)$ & $0.0221(7)$ \\
\hline H51A & 0.7810 & 1.4542 & 0.4438 & $0.033^{*}$ \\
\hline H51B & 0.6528 & 1.3852 & 0.4131 & $0.033 *$ \\
\hline $\mathrm{H} 51 \mathrm{C}$ & 0.5011 & 1.4437 & 0.4551 & $0.033 *$ \\
\hline C52 & $0.5907(5)$ & $1.5130(2)$ & $0.3317(2)$ & 0.0169 (6) \\
\hline H52 & 0.7312 & 1.5129 & 0.2957 & $0.020^{*}$ \\
\hline C53 & $0.3662(6)$ & $1.5002(2)$ & $0.2866(2)$ & $0.0210(7)$ \\
\hline H53A & 0.2261 & 1.5023 & 0.3212 & $0.025^{*}$ \\
\hline H53B & 0.3453 & 1.5488 & 0.2319 & $0.025^{*}$ \\
\hline C54 & $0.3771(6)$ & 1.4147 (2) & $0.2712(2)$ & 0.0199 (7) \\
\hline C55 & $0.1915(6)$ & $1.3571(2)$ & $0.2974(2)$ & $0.0270(8)$ \\
\hline H55 & 0.0617 & 1.3712 & 0.3268 & $0.032 *$ \\
\hline C56 & $0.1967(6)$ & $1.2795(2)$ & $0.2806(2)$ & $0.0302(8)$ \\
\hline H56 & 0.0693 & 1.2416 & 0.2980 & $0.036^{*}$ \\
\hline C57 & $0.3853(6)$ & $1.2574(2)$ & $0.2389(2)$ & $0.0264(8)$ \\
\hline H57 & 0.3864 & 1.2053 & 0.2264 & $0.032 *$ \\
\hline C58 & $0.5734(6)$ & $1.3121(2)$ & $0.2154(2)$ & $0.0234(7)$ \\
\hline H58 & 0.7063 & 1.2961 & 0.1886 & $0.028^{*}$ \\
\hline C59 & $0.5690(6)$ & $1.3899(2)$ & $0.2307(2)$ & $0.0212(7)$ \\
\hline H59 & 0.6982 & 1.4270 & 0.2134 & $0.025^{*}$ \\
\hline C61 & $-0.2773(6)$ & $1.8390(2)$ & -0.0155 (2) & $0.0241(8)$ \\
\hline H61A & -0.2662 & 1.7821 & -0.0225 & $0.036^{*}$ \\
\hline H61B & -0.2750 & 1.8855 & -0.0708 & $0.036^{*}$ \\
\hline $\mathrm{H} 61 \mathrm{C}$ & -0.4257 & 1.8448 & 0.0126 & $0.036^{*}$ \\
\hline C62 & $-0.0668(6)$ & $1.8463(2)$ & $0.0380(2)$ & $0.0181(7)$ \\
\hline H62 & 0.0833 & 1.8338 & 0.0120 & $0.022 *$ \\
\hline C63 & $-0.0560(6)$ & $1.9360(2)$ & $0.0462(2)$ & $0.0202(7)$ \\
\hline $\mathrm{H} 63 \mathrm{~A}$ & -0.2022 & 1.9480 & 0.0734 & $0.024 *$ \\
\hline H63B & 0.0807 & 1.9354 & 0.0831 & $0.024 *$ \\
\hline C64 & $-0.0316(6)$ & $2.0087(2)$ & $-0.0382(2)$ & $0.0187(7)$ \\
\hline C65 & $0.1685(6)$ & $2.0117(2)$ & -0.0867 (3) & $0.0257(8)$ \\
\hline H65 & 0.2927 & 1.9680 & -0.0661 & $0.031 *$ \\
\hline C66 & $0.1908(6)$ & $2.0772(2)$ & $-0.1647(3)$ & $0.0265(9)$ \\
\hline H66 & 0.3303 & 2.0780 & -0.1963 & $0.032 *$ \\
\hline C67 & $0.0122(6)$ & $2.1413(2)$ & $-0.1967(3)$ & $0.0222(8)$ \\
\hline H67 & 0.0275 & 2.1860 & -0.2500 & $0.027 *$ \\
\hline C68 & $-0.1906(6)$ & $2.1390(2)$ & -0.1493 & $0.0231(8)$ \\
\hline H68 & -0.3157 & 2.1821 & -0.1708 & $0.028^{*}$ \\
\hline C69 & $-0.2120(6)$ & $2.0741(2)$ & $-0.0708(2)$ & $0.0219(8)$ \\
\hline H69 & -0.3504 & 2.0740 & -0.0388 & $0.026^{*}$ \\
\hline
\end{tabular}


Atomic displacement parameters $\left(\AA^{2}\right)$ for (I)

\begin{tabular}{|c|c|c|c|c|c|c|}
\hline & $U^{11}$ & $U^{22}$ & $U^{33}$ & $U^{12}$ & $U^{13}$ & $U^{23}$ \\
\hline $\mathrm{Cl1}$ & $0.0149(4)$ & $0.0286(4)$ & $0.0238(4)$ & $-0.0002(3)$ & $0.0020(3)$ & $-0.0123(4)$ \\
\hline $\mathrm{Cl} 2$ & $0.0184(4)$ & $0.0175(4)$ & $0.0292(5)$ & $-0.0041(3)$ & $-0.0007(3)$ & -0.0042 (4) \\
\hline $\mathrm{Cl} 3$ & 0.0159 (4) & $0.0193(4)$ & $0.0221(4)$ & $-0.0018(3)$ & 0.0022 & $-0.0074(3)$ \\
\hline $\mathrm{Cl} 4$ & $0.0146(3)$ & 0.0194 (4) & $0.0205(4)$ & $0.0010(3)$ & -0.0025 & -0.0038 \\
\hline $\mathrm{Cl} 5$ & $0.0218(4)$ & $0.0171(4)$ & $0.0239(4)$ & $-0.0034(3)$ & 0.0064 & -0.0067 (4) \\
\hline $\mathrm{Cl} 6$ & $0.0176(4)$ & $0.0209(4)$ & $0.0259(4)$ & -0.0021 & $0.0013(3)$ & -0.0065 (4) \\
\hline N1 & $0.0214(14)$ & $0.0191(14)$ & $0.0227(16)$ & $0.0005(11)$ & $0.0057(12)$ & $-0.0092(13)$ \\
\hline $\mathrm{N} 2$ & $0.0184(14)$ & $0.0227(15)$ & $0.0293(17)$ & $-0.0036(11)$ & $0.0017(12)$ & $-0.0104(13)$ \\
\hline N3 & $0.0147(13)$ & $0.0219(14)$ & $0.0170(14)$ & $-0.0023(11)$ & $0.0035(11)$ & $-0.0075(12)$ \\
\hline N4 & $0.0147(13)$ & $0.0175(14)$ & $0.0252(16)$ & $-0.0031(10)$ & $-0.0018(12)$ & $-0.0083(12)$ \\
\hline N5 & $0.0137(13)$ & $0.0159(13)$ & 0.0195 (14) & $-0.0023(10)$ & $-0.0036(11)$ & $-0.0028(11)$ \\
\hline N6 & $0.0137(13)$ & $0.0166(13)$ & $0.0226(15)$ & $0.0022(10)$ & $-0.0021(11)$ & $-0.0053(12)$ \\
\hline $\mathrm{C} 1$ & $0.032(2)$ & $0.028(2)$ & $0.034(2)$ & $-0.0059(16)$ & $0.0145(18)$ & $-0.0135(18)$ \\
\hline $\mathrm{C} 2$ & $0.0263(18)$ & $0.0133(15)$ & $0.0204(18)$ & $-0.0026(13)$ & $0.0025(14)$ & $-0.0047(14)$ \\
\hline $\mathrm{C} 3$ & $0.0308(19)$ & $0.0196(17)$ & $0.0169(17)$ & $0.0021(14)$ & $0.0065(14)$ & $-0.0055(14)$ \\
\hline $\mathrm{C} 4$ & $0.0230(18)$ & $0.0187(17)$ & $0.0184(18)$ & $-0.0029(14)$ & $0.0075(15)$ & $-0.0054(14)$ \\
\hline $\mathrm{C} 5$ & $0.0167(16)$ & $0.0210(17)$ & $0.0236(19)$ & $0.0006(13)$ & $0.0034(14)$ & $-0.0030(15)$ \\
\hline C6 & $0.0200(17)$ & $0.0263(19)$ & 0.0244 (19) & $-0.0064(14)$ & $0.0043(14)$ & $-0.0094(16)$ \\
\hline $\mathrm{C} 7$ & $0.0276(18)$ & $0.0171(17)$ & $0.026(2)$ & $-0.0047(14)$ & $0.0118(16)$ & $-0.0115(15)$ \\
\hline $\mathrm{C} 8$ & $0.0225(18)$ & $0.0213(18)$ & $0.030(2)$ & $0.0026(14)$ & $0.0007(16)$ & $-0.0103(16)$ \\
\hline $\mathrm{C} 9$ & $0.0218(17)$ & $0.0197(17)$ & 0.0224 (19) & $-0.0027(14)$ & $0.0014(15)$ & $-0.0067(15)$ \\
\hline $\mathrm{C} 11$ & $0.052(3)$ & $0.022(2)$ & $0.042(3)$ & $-0.0041(18)$ & $0.003(2)$ & $-0.0064(19)$ \\
\hline $\mathrm{C} 12$ & $0.0269(19)$ & $0.035(2)$ & $0.0187(18)$ & $-0.0052(15)$ & $-0.0022(14)$ & $-0.0008(16)$ \\
\hline $\mathrm{C} 13$ & $0.0233(18)$ & $0.036(2)$ & $0.028(2)$ & $-0.0033(15)$ & $0.0006(15)$ & $-0.0084(17)$ \\
\hline $\mathrm{C} 14$ & $0.0292(19)$ & $0.032(2)$ & $0.0250(19)$ & $-0.0063(15)$ & $0.0063(15)$ & $-0.0074(16)$ \\
\hline $\mathrm{C} 15$ & $0.050(3)$ & $0.045(3)$ & $0.039(2)$ & $-0.022(2)$ & $-0.018(2)$ & $0.001(2)$ \\
\hline $\mathrm{C} 16$ & $0.065(3)$ & $0.034(2)$ & $0.045(3)$ & $-0.024(2)$ & $-0.017(2)$ & $-0.004(2)$ \\
\hline $\mathrm{C} 17$ & $0.047(2)$ & 0.0264 (19) & $0.029(2)$ & $0.0000(16)$ & $-0.0053(17)$ & $-0.0091(16)$ \\
\hline $\mathrm{C} 18$ & $0.036(2)$ & $0.0252(19)$ & $0.046(2)$ & $0.0041(15)$ & $-0.0166(18)$ & $-0.0090(18)$ \\
\hline C19 & $0.036(2)$ & 0.0243 (19) & $0.044(2)$ & $-0.0054(16)$ & $-0.0046(18)$ & $-0.0087(18)$ \\
\hline $\mathrm{C} 21$ & $0.0243(18)$ & $0.0213(18)$ & $0.0258(19)$ & $-0.0052(14)$ & $0.0102(15)$ & $-0.0089(15)$ \\
\hline $\mathrm{C} 22$ & $0.0164(15)$ & $0.0149(15)$ & $0.0163(16)$ & $0.0003(12)$ & $0.0004(13)$ & $-0.0063(13)$ \\
\hline $\mathrm{C} 23$ & $0.0258(17)$ & $0.0212(17)$ & $0.0195(18)$ & $-0.0011(13)$ & $0.0060(14)$ & $-0.0052(15)$ \\
\hline $\mathrm{C} 24$ & $0.0252(17)$ & $0.0140(15)$ & $0.0161(16)$ & $-0.0050(12)$ & $0.0053(14)$ & $-0.0020(13)$ \\
\hline $\mathrm{C} 25$ & $0.0191(17)$ & $0.0233(18)$ & $0.035(2)$ & $0.0036(14)$ & $-0.0019(16)$ & $-0.0069(17)$ \\
\hline $\mathrm{C} 26$ & $0.0180(17)$ & 0.0275 (19) & $0.034(2)$ & $-0.0032(14)$ & $-0.0056(15)$ & $-0.0077(17)$ \\
\hline $\mathrm{C} 27$ & $0.0261(18)$ & $0.0172(17)$ & $0.024(2)$ & $-0.0054(14)$ & $0.0044(15)$ & $-0.0044(15)$ \\
\hline $\mathrm{C} 28$ & $0.0272(18)$ & $0.0183(17)$ & $0.031(2)$ & $0.0024(14)$ & $-0.0023(16)$ & $-0.0065(16)$ \\
\hline $\mathrm{C} 29$ & $0.0214(17)$ & $0.0222(17)$ & $0.0235(18)$ & $0.0033(13)$ & $-0.0071(15)$ & $-0.0042(15)$ \\
\hline $\mathrm{C} 31$ & $0.0279(19)$ & $0.029(2)$ & $0.027(2)$ & $-0.0052(16)$ & $-0.0126(16)$ & $-0.0062(17)$ \\
\hline $\mathrm{C} 32$ & $0.0166(15)$ & $0.0174(16)$ & $0.0160(16)$ & $0.0004(12)$ & $-0.0020(14)$ & $-0.0034(14)$ \\
\hline $\mathrm{C} 33$ & $0.0229(17)$ & $0.0184(17)$ & 0.0240 (19) & $0.0016(13)$ & $-0.0013(14)$ & $-0.0091(14)$ \\
\hline C34 & $0.0237(17)$ & $0.0141(16)$ & 0.0232 (19) & $-0.0033(13)$ & $-0.0020(15)$ & $-0.0072(15)$ \\
\hline $\mathrm{C} 35$ & $0.0197(17)$ & $0.0196(18)$ & $0.031(2)$ & $0.0003(14)$ & $-0.0020(15)$ & $-0.0051(16)$ \\
\hline $\mathrm{C} 36$ & $0.0265(19)$ & $0.0261(19)$ & $0.031(2)$ & $-0.0034(15)$ & $0.0041(16)$ & $-0.0087(17)$ \\
\hline $\mathrm{C} 37$ & $0.0303(19)$ & $0.0176(17)$ & $0.024(2)$ & $-0.0077(14)$ & $0.0029(16)$ & $-0.0051(15)$ \\
\hline C38 & $0.0291(19)$ & $0.0161(17)$ & $0.029(2)$ & $0.0020(14)$ & $-0.0066(16)$ & $-0.0070(15)$ \\
\hline C39 & $0.0215(17)$ & $0.0181(17)$ & $0.0256(19)$ & $0.0027(13)$ & $0.0030(14)$ & $-0.0093(15)$ \\
\hline C51 & $0.0283(18)$ & $0.0178(16)$ & $0.0175(17)$ & $0.0020(13)$ & $-0.0015(14)$ & $-0.0030(14)$ \\
\hline C52 & $0.0154(15)$ & $0.0163(15)$ & $0.0186(16)$ & $0.0002(12)$ & $0.0015(12)$ & $-0.0060(13)$ \\
\hline C53 & $0.0213(16)$ & $0.0190(16)$ & $0.0238(18)$ & $0.0003(12)$ & $-0.0024(13)$ & $-0.0089(14)$ \\
\hline
\end{tabular}


supplementary materials

\begin{tabular}{lllllll} 
C54 & $0.0198(16)$ & $0.0241(17)$ & $0.0138(15)$ & $0.0016(13)$ & $-0.0068(13)$ & $-0.0044(13)$ \\
C55 & $0.0155(16)$ & $0.030(2)$ & $0.038(2)$ & $0.0026(14)$ & $-0.0013(15)$ & $-0.0166(17)$ \\
C56 & $0.0223(18)$ & $0.0277(19)$ & $0.043(2)$ & $-0.0042(14)$ & $-0.0064(16)$ & $-0.0151(18)$ \\
C57 & $0.0308(19)$ & $0.0200(17)$ & $0.030(2)$ & $0.0026(14)$ & $-0.0103(16)$ & $-0.0118(15)$ \\
C58 & $0.0292(17)$ & $0.0209(16)$ & $0.0196(17)$ & $0.0050(13)$ & $-0.0034(14)$ & $-0.0074(14)$ \\
C59 & $0.0231(16)$ & $0.0242(17)$ & $0.0160(16)$ & $-0.0005(13)$ & $-0.0016(13)$ & $-0.0067(14)$ \\
C61 & $0.0254(18)$ & $0.0212(18)$ & $0.0227(19)$ & $-0.0039(14)$ & $-0.0039(15)$ & $-0.0038(15)$ \\
C62 & $0.0202(16)$ & $0.0149(16)$ & $0.0173(17)$ & $0.0004(12)$ & $-0.0047(14)$ & $-0.0033(13)$ \\
C63 & $0.0266(18)$ & $0.0129(16)$ & $0.0189(17)$ & $0.0009(13)$ & $-0.0033(14)$ & $-0.0032(13)$ \\
C64 & $0.0205(16)$ & $0.0107(15)$ & $0.0245(19)$ & $0.0004(12)$ & $-0.0031(15)$ & $-0.0056(14)$ \\
C65 & $0.0183(17)$ & $0.0172(17)$ & $0.036(2)$ & $0.0031(13)$ & $-0.0027(16)$ & $-0.0028(16)$ \\
C66 & $0.0209(18)$ & $0.0183(18)$ & $0.039(2)$ & $-0.0041(13)$ & $0.0080(16)$ & $-0.0077(16)$ \\
C67 & $0.0259(19)$ & $0.0143(17)$ & $0.024(2)$ & $-0.0054(13)$ & $0.0019(15)$ & $-0.0033(15)$ \\
C68 & $0.0200(17)$ & $0.0156(17)$ & $0.030(2)$ & $0.0054(13)$ & $-0.0030(15)$ & $-0.0048(15)$ \\
C69 & $0.0218(17)$ & $0.0144(16)$ & $0.030(2)$ & $-0.0026(13)$ & $0.0062(15)$ & $-0.0081(15)$ \\
\hline
\end{tabular}

Geometric parameters $\left(\AA,{ }^{\circ}\right)$ for (I)

\begin{tabular}{|c|c|c|c|}
\hline $\mathrm{N} 1-\mathrm{C} 2$ & $1.515(4)$ & $\mathrm{C} 23-\mathrm{H} 23 \mathrm{~B}$ & 0.9900 \\
\hline $\mathrm{N} 1-\mathrm{H} 1 \mathrm{~A}$ & 0.9100 & $\mathrm{C} 24-\mathrm{C} 29$ & $1.385(5)$ \\
\hline $\mathrm{N} 1-\mathrm{H} 1 \mathrm{~B}$ & 0.9100 & $\mathrm{C} 24-\mathrm{C} 25$ & $1.403(5)$ \\
\hline $\mathrm{N} 1-\mathrm{H} 1 \mathrm{C}$ & 0.9100 & $\mathrm{C} 25-\mathrm{C} 26$ & $1.374(5)$ \\
\hline $\mathrm{N} 2-\mathrm{C} 12$ & $1.531(5)$ & $\mathrm{C} 25-\mathrm{H} 25$ & 0.9500 \\
\hline $\mathrm{N} 2-\mathrm{H} 2 \mathrm{~A}$ & 0.9100 & $\mathrm{C} 26-\mathrm{C} 27$ & $1.391(5)$ \\
\hline $\mathrm{N} 2-\mathrm{H} 2 \mathrm{~B}$ & 0.9100 & $\mathrm{C} 26-\mathrm{H} 26$ & 0.9500 \\
\hline $\mathrm{N} 2-\mathrm{H} 2 \mathrm{C}$ & 0.9100 & $\mathrm{C} 27-\mathrm{C} 28$ & $1.385(5)$ \\
\hline N3-C22 & $1.494(4)$ & $\mathrm{C} 27-\mathrm{H} 27$ & 0.9500 \\
\hline N3- H3A & 0.9100 & $\mathrm{C} 28-\mathrm{C} 29$ & $1.403(5)$ \\
\hline N3-H3B & 0.9100 & $\mathrm{C} 28-\mathrm{H} 28$ & 0.9500 \\
\hline $\mathrm{N} 3-\mathrm{H} 3 \mathrm{C}$ & 0.9100 & $\mathrm{C} 29-\mathrm{H} 29$ & 0.9500 \\
\hline $\mathrm{N} 4-\mathrm{C} 32$ & $1.497(4)$ & $\mathrm{C} 31-\mathrm{C} 32$ & $1.537(5)$ \\
\hline $\mathrm{N} 4-\mathrm{H} 4 \mathrm{~A}$ & 0.9100 & $\mathrm{C} 31-\mathrm{H} 31 \mathrm{~A}$ & 0.9800 \\
\hline $\mathrm{N} 4-\mathrm{H} 4 \mathrm{~B}$ & 0.9100 & $\mathrm{C} 31-\mathrm{H} 31 \mathrm{~B}$ & 0.9800 \\
\hline $\mathrm{N} 4-\mathrm{H} 4 \mathrm{C}$ & 0.9100 & $\mathrm{C} 31-\mathrm{H} 31 \mathrm{C}$ & 0.9800 \\
\hline N5-C52 & $1.508(4)$ & $\mathrm{C} 32-\mathrm{C} 33$ & $1.525(4)$ \\
\hline N5- $\mathrm{H} 5 \mathrm{~A}$ & 0.9100 & C $32-\mathrm{H} 32$ & 1.0000 \\
\hline N5- H5B & 0.9100 & $\mathrm{C} 33-\mathrm{C} 34$ & $1.511(5)$ \\
\hline N5- $\mathrm{H} 5 \mathrm{C}$ & 0.9100 & $\mathrm{C} 33-\mathrm{H} 33 \mathrm{~A}$ & 0.9900 \\
\hline N6-C62 & $1.495(4)$ & С $33-\mathrm{H} 33 \mathrm{~B}$ & 0.9900 \\
\hline N6-H6A & 0.9100 & C34-C35 & $1.394(5)$ \\
\hline N6-H6B & 0.9100 & C34-C39 & $1.395(5)$ \\
\hline N6- $\mathrm{H} 6 \mathrm{C}$ & 0.9100 & $\mathrm{C} 35-\mathrm{C} 36$ & $1.385(5)$ \\
\hline $\mathrm{C} 1-\mathrm{C} 2$ & $1.505(5)$ & C $35-\mathrm{H} 35$ & 0.9500 \\
\hline $\mathrm{C} 1-\mathrm{H} 1 \mathrm{D}$ & 0.9800 & $\mathrm{C} 36-\mathrm{C} 37$ & $1.395(5)$ \\
\hline $\mathrm{C} 1-\mathrm{H} 1 \mathrm{E}$ & 0.9800 & $\mathrm{C} 36-\mathrm{H} 36$ & 0.9500 \\
\hline $\mathrm{C} 1-\mathrm{H} 1 \mathrm{~F}$ & 0.9800 & $\mathrm{C} 37-\mathrm{C} 38$ & $1.389(5)$ \\
\hline $\mathrm{C} 2-\mathrm{C} 3$ & $1.537(4)$ & C37-H37 & 0.9500 \\
\hline $\mathrm{C} 2-\mathrm{H} 2$ & 1.0000 & C $38-\mathrm{C} 39$ & $1.397(5)$ \\
\hline $\mathrm{C} 3-\mathrm{C} 4$ & $1.514(5)$ & C $38-\mathrm{H} 38$ & 0.9500 \\
\hline C3-H3D & 0.9900 & С $39-\mathrm{H} 39$ & 0.9500 \\
\hline $\mathrm{C} 3-\mathrm{H} 3 \mathrm{E}$ & 0.9900 & C51-C52 & $1.534(4)$ \\
\hline $\mathrm{C} 4-\mathrm{C} 5$ & $1.390(5)$ & C51-H51A & 0.9800 \\
\hline $\mathrm{C} 4-\mathrm{C} 9$ & $1.404(5)$ & С51-H51B & 0.9800 \\
\hline $\mathrm{C} 5-\mathrm{C} 6$ & $1.391(5)$ & $\mathrm{C} 51-\mathrm{H} 51 \mathrm{C}$ & 0.9800 \\
\hline
\end{tabular}


supplementary materials

\begin{tabular}{|c|c|c|c|}
\hline $\mathrm{C} 5-\mathrm{H} 5$ & 0.9500 & $\mathrm{C} 52-\mathrm{C} 53$ & $1.539(4)$ \\
\hline $\mathrm{C} 6-\mathrm{C} 7$ & $1.394(5)$ & C52-H52 & 1.0000 \\
\hline C6-H6 & 0.9500 & C53-C54 & $1.511(5)$ \\
\hline $\mathrm{C} 7-\mathrm{C} 8$ & $1.378(6)$ & $\mathrm{C} 53-\mathrm{H} 53 \mathrm{~A}$ & 0.9900 \\
\hline $\mathrm{C} 7-\mathrm{H} 7$ & 0.9500 & С53-H53B & 0.9900 \\
\hline $\mathrm{C} 8-\mathrm{C} 9$ & $1.382(5)$ & C54-C59 & $1.401(5)$ \\
\hline $\mathrm{C} 8-\mathrm{H} 8$ & 0.9500 & C54-C55 & $1.408(5)$ \\
\hline C9- $\mathrm{H} 9$ & 0.9500 & $\mathrm{C} 55-\mathrm{C} 56$ & $1.397(5)$ \\
\hline $\mathrm{C} 11-\mathrm{C} 12$ & $1.504(5)$ & $\mathrm{C} 55-\mathrm{H} 55$ & 0.9500 \\
\hline C11-H11A & 0.9800 & $\mathrm{C} 56-\mathrm{C} 57$ & $1.381(5)$ \\
\hline $\mathrm{C} 11-\mathrm{H} 11 \mathrm{~B}$ & 0.9800 & C56- $-\mathrm{H} 56$ & 0.9500 \\
\hline $\mathrm{C} 11-\mathrm{H} 11 \mathrm{C}$ & 0.9800 & $\mathrm{C} 57-\mathrm{C} 58$ & $1.388(5)$ \\
\hline $\mathrm{C} 12-\mathrm{C} 13$ & $1.521(5)$ & C57-H57 & 0.9500 \\
\hline $\mathrm{C} 12-\mathrm{H} 12$ & 1.0000 & $\mathrm{C} 58-\mathrm{C} 59$ & $1.388(5)$ \\
\hline $\mathrm{C} 13-\mathrm{C} 14$ & $1.543(5)$ & C58- $\mathrm{H} 58$ & 0.9500 \\
\hline $\mathrm{C} 13-\mathrm{H} 13 \mathrm{~A}$ & 0.9900 & C59-H59 & 0.9500 \\
\hline $\mathrm{C} 13-\mathrm{H} 13 \mathrm{~B}$ & 0.9900 & $\mathrm{C} 61-\mathrm{C} 62$ & $1.528(5)$ \\
\hline $\mathrm{C} 14-\mathrm{C} 15$ & $1.385(5)$ & C61-H61A & 0.9800 \\
\hline $\mathrm{C} 14-\mathrm{C} 19$ & $1.393(5)$ & C61-H61B & 0.9800 \\
\hline $\mathrm{C} 15-\mathrm{C} 16$ & $1.370(6)$ & C61-H61C & 0.9800 \\
\hline C15-H15 & 0.9500 & $\mathrm{C} 62-\mathrm{C} 63$ & $1.529(4)$ \\
\hline $\mathrm{C} 16-\mathrm{C} 17$ & $1.374(6)$ & C62-H62 & 1.0000 \\
\hline $\mathrm{C} 16-\mathrm{H} 16$ & 0.9500 & C63-C64 & $1.518(5)$ \\
\hline $\mathrm{C} 17-\mathrm{C} 18$ & $1.369(5)$ & C63-H63A & 0.9900 \\
\hline C17-H17 & 0.9500 & С63-H63B & 0.9900 \\
\hline $\mathrm{C} 18-\mathrm{C} 19$ & $1.380(5)$ & C64-C65 & $1.389(5)$ \\
\hline $\mathrm{C} 18-\mathrm{H} 18$ & 0.9500 & C64-C69 & $1.403(5)$ \\
\hline C19-H19 & 0.9500 & $\mathrm{C} 65-\mathrm{C} 66$ & $1.391(5)$ \\
\hline $\mathrm{C} 21-\mathrm{C} 22$ & $1.511(5)$ & C65-H65 & 0.9500 \\
\hline $\mathrm{C} 21-\mathrm{H} 21 \mathrm{~A}$ & 0.9800 & $\mathrm{C} 66-\mathrm{C} 67$ & $1.382(5)$ \\
\hline $\mathrm{C} 21-\mathrm{H} 21 \mathrm{~B}$ & 0.9800 & C66-H66 & 0.9500 \\
\hline $\mathrm{C} 21-\mathrm{H} 21 \mathrm{C}$ & 0.9800 & $\mathrm{C} 67-\mathrm{C} 68$ & $1.392(5)$ \\
\hline $\mathrm{C} 22-\mathrm{C} 23$ & $1.542(4)$ & C67-H67 & 0.9500 \\
\hline $\mathrm{C} 22-\mathrm{H} 22$ & 1.0000 & $\mathrm{C} 68-\mathrm{C} 69$ & $1.392(5)$ \\
\hline $\mathrm{C} 23-\mathrm{C} 24$ & $1.505(5)$ & C68-H68 & 0.9500 \\
\hline $\mathrm{C} 23-\mathrm{H} 23 \mathrm{~A}$ & 0.9900 & C69-H69 & 0.9500 \\
\hline $\mathrm{C} 2-\mathrm{N} 1-\mathrm{H} 1 \mathrm{~A}$ & 109.5 & $\mathrm{C} 29-\mathrm{C} 24-\mathrm{C} 25$ & $116.9(3)$ \\
\hline $\mathrm{C} 2-\mathrm{N} 1-\mathrm{H} 1 \mathrm{~B}$ & 109.5 & $\mathrm{C} 29-\mathrm{C} 24-\mathrm{C} 23$ & $121.3(3)$ \\
\hline $\mathrm{H} 1 \mathrm{~A}-\mathrm{N} 1-\mathrm{H} 1 \mathrm{~B}$ & 109.5 & $\mathrm{C} 25-\mathrm{C} 24-\mathrm{C} 23$ & $121.8(3)$ \\
\hline $\mathrm{C} 2-\mathrm{N} 1-\mathrm{H} 1 \mathrm{C}$ & 109.5 & $\mathrm{C} 26-\mathrm{C} 25-\mathrm{C} 24$ & $122.3(3)$ \\
\hline $\mathrm{H} 1 \mathrm{~A}-\mathrm{N} 1-\mathrm{H} 1 \mathrm{C}$ & 109.5 & $\mathrm{C} 26-\mathrm{C} 25-\mathrm{H} 25$ & 118.8 \\
\hline $\mathrm{H} 1 \mathrm{~B}-\mathrm{N} 1-\mathrm{H} 1 \mathrm{C}$ & 109.5 & $\mathrm{C} 24-\mathrm{C} 25-\mathrm{H} 25$ & 118.8 \\
\hline $\mathrm{C} 12-\mathrm{N} 2-\mathrm{H} 2 \mathrm{~A}$ & 109.5 & $\mathrm{C} 25-\mathrm{C} 26-\mathrm{C} 27$ & $119.9(3)$ \\
\hline $\mathrm{C} 12-\mathrm{N} 2-\mathrm{H} 2 \mathrm{~B}$ & 109.5 & $\mathrm{C} 25-\mathrm{C} 26-\mathrm{H} 26$ & 120.0 \\
\hline $\mathrm{H} 2 \mathrm{~A}-\mathrm{N} 2-\mathrm{H} 2 \mathrm{~B}$ & 109.5 & $\mathrm{C} 27-\mathrm{C} 26-\mathrm{H} 26$ & 120.0 \\
\hline $\mathrm{C} 12-\mathrm{N} 2-\mathrm{H} 2 \mathrm{C}$ & 109.5 & $\mathrm{C} 28-\mathrm{C} 27-\mathrm{C} 26$ & $119.4(4)$ \\
\hline $\mathrm{H} 2 \mathrm{~A}-\mathrm{N} 2-\mathrm{H} 2 \mathrm{C}$ & 109.5 & $\mathrm{C} 28-\mathrm{C} 27-\mathrm{H} 27$ & 120.3 \\
\hline $\mathrm{H} 2 \mathrm{~B}-\mathrm{N} 2-\mathrm{H} 2 \mathrm{C}$ & 109.5 & $\mathrm{C} 26-\mathrm{C} 27-\mathrm{H} 27$ & 120.3 \\
\hline $\mathrm{C} 22-\mathrm{N} 3-\mathrm{H} 3 \mathrm{~A}$ & 109.5 & $\mathrm{C} 27-\mathrm{C} 28-\mathrm{C} 29$ & $119.8(3)$ \\
\hline $\mathrm{C} 22-\mathrm{N} 3-\mathrm{H} 3 \mathrm{~B}$ & 109.5 & $\mathrm{C} 27-\mathrm{C} 28-\mathrm{H} 28$ & 120.1 \\
\hline $\mathrm{H} 3 \mathrm{~A}-\mathrm{N} 3-\mathrm{H} 3 \mathrm{~B}$ & 109.5 & $\mathrm{C} 29-\mathrm{C} 28-\mathrm{H} 28$ & 120.1 \\
\hline $\mathrm{C} 22-\mathrm{N} 3-\mathrm{H} 3 \mathrm{C}$ & 109.5 & $\mathrm{C} 24-\mathrm{C} 29-\mathrm{C} 28$ & $121.7(3)$ \\
\hline $\mathrm{H} 3 \mathrm{~A}-\mathrm{N} 3-\mathrm{H} 3 \mathrm{C}$ & 109.5 & $\mathrm{C} 24-\mathrm{C} 29-\mathrm{H} 29$ & 119.2 \\
\hline
\end{tabular}




\begin{tabular}{|c|c|c|c|}
\hline $\mathrm{H} 3 \mathrm{~B}-\mathrm{N} 3-\mathrm{H} 3 \mathrm{C}$ & 109.5 & $\mathrm{C} 28-\mathrm{C} 29-\mathrm{H} 29$ & 119.2 \\
\hline $\mathrm{C} 32-\mathrm{N} 4-\mathrm{H} 4 \mathrm{~A}$ & 109.5 & $\mathrm{C} 32-\mathrm{C} 31-\mathrm{H} 31 \mathrm{~A}$ & 109.5 \\
\hline $\mathrm{C} 32-\mathrm{N} 4-\mathrm{H} 4 \mathrm{~B}$ & 109.5 & $\mathrm{C} 32-\mathrm{C} 31-\mathrm{H} 31 \mathrm{~B}$ & 109.5 \\
\hline $\mathrm{H} 4 \mathrm{~A}-\mathrm{N} 4-\mathrm{H} 4 \mathrm{~B}$ & 109.5 & $\mathrm{H} 31 \mathrm{~A}-\mathrm{C} 31-\mathrm{H} 31 \mathrm{~B}$ & 109.5 \\
\hline $\mathrm{C} 32-\mathrm{N} 4-\mathrm{H} 4 \mathrm{C}$ & 109.5 & $\mathrm{C} 32-\mathrm{C} 31-\mathrm{H} 31 \mathrm{C}$ & 109.5 \\
\hline $\mathrm{H} 4 \mathrm{~A}-\mathrm{N} 4-\mathrm{H} 4 \mathrm{C}$ & 109.5 & $\mathrm{H} 31 \mathrm{~A}-\mathrm{C} 31-\mathrm{H} 31 \mathrm{C}$ & 109.5 \\
\hline $\mathrm{H} 4 \mathrm{~B}-\mathrm{N} 4-\mathrm{H} 4 \mathrm{C}$ & 109.5 & $\mathrm{H} 31 \mathrm{~B}-\mathrm{C} 31-\mathrm{H} 31 \mathrm{C}$ & 109.5 \\
\hline $\mathrm{C} 52-\mathrm{N} 5-\mathrm{H} 5 \mathrm{~A}$ & 109.5 & $\mathrm{~N} 4-\mathrm{C} 32-\mathrm{C} 33$ & $110.3(3)$ \\
\hline $\mathrm{C} 52-\mathrm{N} 5-\mathrm{H} 5 \mathrm{~B}$ & 109.5 & $\mathrm{~N} 4-\mathrm{C} 32-\mathrm{C} 31$ & $107.9(3)$ \\
\hline $\mathrm{H} 5 \mathrm{~A}-\mathrm{N} 5-\mathrm{H} 5 \mathrm{~B}$ & 109.5 & $\mathrm{C} 33-\mathrm{C} 32-\mathrm{C} 31$ & $113.3(3)$ \\
\hline $\mathrm{C} 52-\mathrm{N} 5-\mathrm{H} 5 \mathrm{C}$ & 109.5 & $\mathrm{~N} 4-\mathrm{C} 32-\mathrm{H} 32$ & 108.4 \\
\hline $\mathrm{H} 5 \mathrm{~A}-\mathrm{N} 5-\mathrm{H} 5 \mathrm{C}$ & 109.5 & $\mathrm{C} 33-\mathrm{C} 32-\mathrm{H} 32$ & 108.4 \\
\hline $\mathrm{H} 5 \mathrm{~B}-\mathrm{N} 5-\mathrm{H} 5 \mathrm{C}$ & 109.5 & $\mathrm{C} 31-\mathrm{C} 32-\mathrm{H} 32$ & 108.4 \\
\hline $\mathrm{C} 62-\mathrm{N} 6-\mathrm{H} 6 \mathrm{~A}$ & 109.5 & $\mathrm{C} 34-\mathrm{C} 33-\mathrm{C} 32$ & $111.7(3)$ \\
\hline $\mathrm{C} 62-\mathrm{N} 6-\mathrm{H} 6 \mathrm{~B}$ & 109.5 & $\mathrm{C} 34-\mathrm{C} 33-\mathrm{H} 33 \mathrm{~A}$ & 109.3 \\
\hline $\mathrm{H} 6 \mathrm{~A}-\mathrm{N} 6-\mathrm{H} 6 \mathrm{~B}$ & 109.5 & $\mathrm{C} 32-\mathrm{C} 33-\mathrm{H} 33 \mathrm{~A}$ & 109.3 \\
\hline $\mathrm{C} 62-\mathrm{N} 6-\mathrm{H} 6 \mathrm{C}$ & 109.5 & $\mathrm{C} 34-\mathrm{C} 33-\mathrm{H} 33 \mathrm{~B}$ & 109.3 \\
\hline $\mathrm{H} 6 \mathrm{~A}-\mathrm{N} 6-\mathrm{H} 6 \mathrm{C}$ & 109.5 & $\mathrm{C} 32-\mathrm{C} 33-\mathrm{H} 33 \mathrm{~B}$ & 109.3 \\
\hline $\mathrm{H} 6 \mathrm{~B}-\mathrm{N} 6-\mathrm{H} 6 \mathrm{C}$ & 109.5 & $\mathrm{H} 33 \mathrm{~A}-\mathrm{C} 33-\mathrm{H} 33 \mathrm{~B}$ & 107.9 \\
\hline $\mathrm{C} 2-\mathrm{C} 1-\mathrm{H} 1 \mathrm{D}$ & 109.5 & $\mathrm{C} 35-\mathrm{C} 34-\mathrm{C} 39$ & $117.8(3)$ \\
\hline $\mathrm{C} 2-\mathrm{C} 1-\mathrm{H} 1 \mathrm{E}$ & 109.5 & $\mathrm{C} 35-\mathrm{C} 34-\mathrm{C} 33$ & $120.8(3)$ \\
\hline $\mathrm{H} 1 \mathrm{D}-\mathrm{C} 1-\mathrm{H} 1 \mathrm{E}$ & 109.5 & $\mathrm{C} 39-\mathrm{C} 34-\mathrm{C} 33$ & $121.2(3)$ \\
\hline $\mathrm{C} 2-\mathrm{C} 1-\mathrm{H} 1 \mathrm{~F}$ & 109.5 & $\mathrm{C} 36-\mathrm{C} 35-\mathrm{C} 34$ & $121.1(3)$ \\
\hline $\mathrm{H} 1 \mathrm{D}-\mathrm{C} 1-\mathrm{H} 1 \mathrm{~F}$ & 109.5 & $\mathrm{C} 36-\mathrm{C} 35-\mathrm{H} 35$ & 119.4 \\
\hline $\mathrm{H} 1 \mathrm{E}-\mathrm{C} 1-\mathrm{H} 1 \mathrm{~F}$ & 109.5 & $\mathrm{C} 34-\mathrm{C} 35-\mathrm{H} 35$ & 119.4 \\
\hline $\mathrm{C} 1-\mathrm{C} 2-\mathrm{N} 1$ & $107.8(3)$ & $\mathrm{C} 35-\mathrm{C} 36-\mathrm{C} 37$ & $120.6(3)$ \\
\hline $\mathrm{C} 1-\mathrm{C} 2-\mathrm{C} 3$ & $114.1(3)$ & $\mathrm{C} 35-\mathrm{C} 36-\mathrm{H} 36$ & 119.7 \\
\hline $\mathrm{N} 1-\mathrm{C} 2-\mathrm{C} 3$ & $108.1(3)$ & $\mathrm{C} 37-\mathrm{C} 36-\mathrm{H} 36$ & 119.7 \\
\hline $\mathrm{C} 1-\mathrm{C} 2-\mathrm{H} 2$ & 108.9 & $\mathrm{C} 38-\mathrm{C} 37-\mathrm{C} 36$ & $119.1(4)$ \\
\hline $\mathrm{N} 1-\mathrm{C} 2-\mathrm{H} 2$ & 108.9 & $\mathrm{C} 38-\mathrm{C} 37-\mathrm{H} 37$ & 120.4 \\
\hline $\mathrm{C} 3-\mathrm{C} 2-\mathrm{H} 2$ & 108.9 & $\mathrm{C} 36-\mathrm{C} 37-\mathrm{H} 37$ & 120.4 \\
\hline $\mathrm{C} 4-\mathrm{C} 3-\mathrm{C} 2$ & $112.0(3)$ & $\mathrm{C} 37-\mathrm{C} 38-\mathrm{C} 39$ & $119.8(3)$ \\
\hline $\mathrm{C} 4-\mathrm{C} 3-\mathrm{H} 3 \mathrm{D}$ & 109.2 & $\mathrm{C} 37-\mathrm{C} 38-\mathrm{H} 38$ & 120.1 \\
\hline $\mathrm{C} 2-\mathrm{C} 3-\mathrm{H} 3 \mathrm{D}$ & 109.2 & $\mathrm{C} 39-\mathrm{C} 38-\mathrm{H} 38$ & 120.1 \\
\hline $\mathrm{C} 4-\mathrm{C} 3-\mathrm{H} 3 \mathrm{E}$ & 109.2 & $\mathrm{C} 34-\mathrm{C} 39-\mathrm{C} 38$ & $121.5(3)$ \\
\hline $\mathrm{C} 2-\mathrm{C} 3-\mathrm{H} 3 \mathrm{E}$ & 109.2 & C34-C39-H39 & 119.3 \\
\hline $\mathrm{H} 3 \mathrm{D}-\mathrm{C} 3-\mathrm{H} 3 \mathrm{E}$ & 107.9 & $\mathrm{C} 38-\mathrm{C} 39-\mathrm{H} 39$ & 119.3 \\
\hline $\mathrm{C} 5-\mathrm{C} 4-\mathrm{C} 9$ & $118.6(3)$ & $\mathrm{C} 52-\mathrm{C} 51-\mathrm{H} 51 \mathrm{~A}$ & 109.5 \\
\hline $\mathrm{C} 5-\mathrm{C} 4-\mathrm{C} 3$ & $121.1(3)$ & $\mathrm{C} 52-\mathrm{C} 51-\mathrm{H} 51 \mathrm{~B}$ & 109.5 \\
\hline $\mathrm{C} 9-\mathrm{C} 4-\mathrm{C} 3$ & $120.2(3)$ & $\mathrm{H} 51 \mathrm{~A}-\mathrm{C} 51-\mathrm{H} 51 \mathrm{~B}$ & 109.5 \\
\hline $\mathrm{C} 4-\mathrm{C} 5-\mathrm{C} 6$ & $120.5(3)$ & $\mathrm{C} 52-\mathrm{C} 51-\mathrm{H} 51 \mathrm{C}$ & 109.5 \\
\hline $\mathrm{C} 4-\mathrm{C} 5-\mathrm{H} 5$ & 119.7 & $\mathrm{H} 51 \mathrm{~A}-\mathrm{C} 51-\mathrm{H} 51 \mathrm{C}$ & 109.5 \\
\hline $\mathrm{C} 6-\mathrm{C} 5-\mathrm{H} 5$ & 119.7 & $\mathrm{H} 51 \mathrm{~B}-\mathrm{C} 51-\mathrm{H} 51 \mathrm{C}$ & 109.5 \\
\hline $\mathrm{C} 5-\mathrm{C} 6-\mathrm{C} 7$ & $120.4(3)$ & $\mathrm{N} 5-\mathrm{C} 52-\mathrm{C} 51$ & $107.9(3)$ \\
\hline $\mathrm{C} 5-\mathrm{C} 6-\mathrm{H} 6$ & 119.8 & $\mathrm{~N} 5-\mathrm{C} 52-\mathrm{C} 53$ & $108.6(2)$ \\
\hline $\mathrm{C} 7-\mathrm{C} 6-\mathrm{H} 6$ & 119.8 & $\mathrm{C} 51-\mathrm{C} 52-\mathrm{C} 53$ & $113.5(3)$ \\
\hline $\mathrm{C} 8-\mathrm{C} 7-\mathrm{C} 6$ & $119.2(4)$ & $\mathrm{N} 5-\mathrm{C} 52-\mathrm{H} 52$ & 108.9 \\
\hline $\mathrm{C} 8-\mathrm{C} 7-\mathrm{H} 7$ & 120.4 & $\mathrm{C} 51-\mathrm{C} 52-\mathrm{H} 52$ & 108.9 \\
\hline $\mathrm{C} 6-\mathrm{C} 7-\mathrm{H} 7$ & 120.4 & $\mathrm{C} 53-\mathrm{C} 52-\mathrm{H} 52$ & 108.9 \\
\hline $\mathrm{C} 7-\mathrm{C} 8-\mathrm{C} 9$ & $120.9(3)$ & $\mathrm{C} 54-\mathrm{C} 53-\mathrm{C} 52$ & $112.6(3)$ \\
\hline $\mathrm{C} 7-\mathrm{C} 8-\mathrm{H} 8$ & 119.6 & $\mathrm{C} 54-\mathrm{C} 53-\mathrm{H} 53 \mathrm{~A}$ & 109.1 \\
\hline $\mathrm{C} 9-\mathrm{C} 8-\mathrm{H} 8$ & 119.6 & $\mathrm{C} 52-\mathrm{C} 53-\mathrm{H} 53 \mathrm{~A}$ & 109.1 \\
\hline $\mathrm{C} 8-\mathrm{C} 9-\mathrm{C} 4$ & $120.5(3)$ & $\mathrm{C} 54-\mathrm{C} 53-\mathrm{H} 53 \mathrm{~B}$ & 109.1 \\
\hline $\mathrm{C} 8-\mathrm{C} 9-\mathrm{H} 9$ & 119.8 & $\mathrm{C} 52-\mathrm{C} 53-\mathrm{H} 53 \mathrm{~B}$ & 109.1 \\
\hline
\end{tabular}




\section{supplementary materials}

\begin{tabular}{|c|c|c|c|}
\hline $\mathrm{C} 4-\mathrm{C} 9-\mathrm{H} 9$ & 119.8 & $\mathrm{H} 53 \mathrm{~A}-\mathrm{C} 53-\mathrm{H} 53 \mathrm{~B}$ & 107.8 \\
\hline $\mathrm{C} 12-\mathrm{C} 11-\mathrm{H} 11 \mathrm{~A}$ & 109.5 & C59-C54-C55 & $117.7(3)$ \\
\hline $\mathrm{C} 12-\mathrm{C} 11-\mathrm{H} 11 \mathrm{~B}$ & 109.5 & $\mathrm{C} 59-\mathrm{C} 54-\mathrm{C} 53$ & $121.9(3)$ \\
\hline $\mathrm{H} 11 \mathrm{~A}-\mathrm{C} 11-\mathrm{H} 11 \mathrm{~B}$ & 109.5 & $\mathrm{C} 55-\mathrm{C} 54-\mathrm{C} 53$ & $120.4(3)$ \\
\hline $\mathrm{C} 12-\mathrm{C} 11-\mathrm{H} 11 \mathrm{C}$ & 109.5 & $\mathrm{C} 56-\mathrm{C} 55-\mathrm{C} 54$ & $120.6(3)$ \\
\hline $\mathrm{H} 11 \mathrm{~A}-\mathrm{C} 11-\mathrm{H} 11 \mathrm{C}$ & 109.5 & $\mathrm{C} 56-\mathrm{C} 55-\mathrm{H} 55$ & 119.7 \\
\hline $\mathrm{H} 11 \mathrm{~B}-\mathrm{C} 11-\mathrm{H} 11 \mathrm{C}$ & 109.5 & $\mathrm{C} 54-\mathrm{C} 55-\mathrm{H} 55$ & 119.7 \\
\hline $\mathrm{C} 11-\mathrm{C} 12-\mathrm{C} 13$ & $113.4(3)$ & $\mathrm{C} 57-\mathrm{C} 56-\mathrm{C} 55$ & $120.6(3)$ \\
\hline $\mathrm{C} 11-\mathrm{C} 12-\mathrm{N} 2$ & $109.1(3)$ & C57-C56-H56 & 119.7 \\
\hline $\mathrm{C} 13-\mathrm{C} 12-\mathrm{N} 2$ & $110.1(3)$ & $\mathrm{C} 55-\mathrm{C} 56-\mathrm{H} 56$ & 119.7 \\
\hline $\mathrm{C} 11-\mathrm{C} 12-\mathrm{H} 12$ & 108.0 & C56-C57-C58 & $119.4(3)$ \\
\hline $\mathrm{C} 13-\mathrm{C} 12-\mathrm{H} 12$ & 108.0 & $\mathrm{C} 56-\mathrm{C} 57-\mathrm{H} 57$ & 120.3 \\
\hline $\mathrm{N} 2-\mathrm{C} 12-\mathrm{H} 12$ & 108.0 & $\mathrm{C} 58-\mathrm{C} 57-\mathrm{H} 57$ & 120.3 \\
\hline $\mathrm{C} 12-\mathrm{C} 13-\mathrm{C} 14$ & $110.6(3)$ & $\mathrm{C} 59-\mathrm{C} 58-\mathrm{C} 57$ & $120.6(3)$ \\
\hline $\mathrm{C} 12-\mathrm{C} 13-\mathrm{H} 13 \mathrm{~A}$ & 109.5 & $\mathrm{C} 59-\mathrm{C} 58-\mathrm{H} 58$ & 119.7 \\
\hline $\mathrm{C} 14-\mathrm{C} 13-\mathrm{H} 13 \mathrm{~A}$ & 109.5 & C57-C58-H58 & 119.7 \\
\hline $\mathrm{C} 12-\mathrm{C} 13-\mathrm{H} 13 \mathrm{~B}$ & 109.5 & $\mathrm{C} 58-\mathrm{C} 59-\mathrm{C} 54$ & $121.1(3)$ \\
\hline $\mathrm{C} 14-\mathrm{C} 13-\mathrm{H} 13 \mathrm{~B}$ & 109.5 & $\mathrm{C} 58-\mathrm{C} 59-\mathrm{H} 59$ & 119.5 \\
\hline $\mathrm{H} 13 \mathrm{~A}-\mathrm{C} 13-\mathrm{H} 13 \mathrm{~B}$ & 108.1 & $\mathrm{C} 54-\mathrm{C} 59-\mathrm{H} 59$ & 119.5 \\
\hline $\mathrm{C} 15-\mathrm{C} 14-\mathrm{C} 19$ & $118.5(4)$ & $\mathrm{C} 62-\mathrm{C} 61-\mathrm{H} 61 \mathrm{~A}$ & 109.5 \\
\hline $\mathrm{C} 15-\mathrm{C} 14-\mathrm{C} 13$ & $121.3(4)$ & $\mathrm{C} 62-\mathrm{C} 61-\mathrm{H} 61 \mathrm{~B}$ & 109.5 \\
\hline $\mathrm{C} 19-\mathrm{C} 14-\mathrm{C} 13$ & $120.2(3)$ & $\mathrm{H} 61 \mathrm{~A}-\mathrm{C} 61-\mathrm{H} 61 \mathrm{~B}$ & 109.5 \\
\hline $\mathrm{C} 16-\mathrm{C} 15-\mathrm{C} 14$ & $120.3(4)$ & C62- $61-\mathrm{H} 61 \mathrm{C}$ & 109.5 \\
\hline $\mathrm{C} 16-\mathrm{C} 15-\mathrm{H} 15$ & 119.9 & $\mathrm{H} 61 \mathrm{~A}-\mathrm{C} 61-\mathrm{H} 61 \mathrm{C}$ & 109.5 \\
\hline $\mathrm{C} 14-\mathrm{C} 15-\mathrm{H} 15$ & 119.9 & $\mathrm{H} 61 \mathrm{~B}-\mathrm{C} 61-\mathrm{H} 61 \mathrm{C}$ & 109.5 \\
\hline $\mathrm{C} 15-\mathrm{C} 16-\mathrm{C} 17$ & $121.0(4)$ & $\mathrm{N} 6-\mathrm{C} 62-\mathrm{C} 61$ & $108.3(3)$ \\
\hline $\mathrm{C} 15-\mathrm{C} 16-\mathrm{H} 16$ & 119.5 & $\mathrm{~N} 6-\mathrm{C} 62-\mathrm{C} 63$ & $108.9(3)$ \\
\hline $\mathrm{C} 17-\mathrm{C} 16-\mathrm{H} 16$ & 119.5 & $\mathrm{C} 61-\mathrm{C} 62-\mathrm{C} 63$ & $113.5(3)$ \\
\hline $\mathrm{C} 18-\mathrm{C} 17-\mathrm{C} 16$ & $119.5(4)$ & $\mathrm{N} 6-\mathrm{C} 62-\mathrm{H} 62$ & 108.7 \\
\hline $\mathrm{C} 18-\mathrm{C} 17-\mathrm{H} 17$ & 120.2 & C61-C62-H62 & 108.7 \\
\hline $\mathrm{C} 16-\mathrm{C} 17-\mathrm{H} 17$ & 120.2 & $\mathrm{C} 63-\mathrm{C} 62-\mathrm{H} 62$ & 108.7 \\
\hline $\mathrm{C} 17-\mathrm{C} 18-\mathrm{C} 19$ & $120.1(4)$ & C64-C63-C62 & $112.7(3)$ \\
\hline $\mathrm{C} 17-\mathrm{C} 18-\mathrm{H} 18$ & 120.0 & $\mathrm{C} 64-\mathrm{C} 63-\mathrm{H} 63 \mathrm{~A}$ & 109.1 \\
\hline $\mathrm{C} 19-\mathrm{C} 18-\mathrm{H} 18$ & 120.0 & $\mathrm{C} 62-\mathrm{C} 63-\mathrm{H} 63 \mathrm{~A}$ & 109.1 \\
\hline $\mathrm{C} 18-\mathrm{C} 19-\mathrm{C} 14$ & $120.6(4)$ & $\mathrm{C} 64-\mathrm{C} 63-\mathrm{H} 63 \mathrm{~B}$ & 109.1 \\
\hline $\mathrm{C} 18-\mathrm{C} 19-\mathrm{H} 19$ & 119.7 & $\mathrm{C} 62-\mathrm{C} 63-\mathrm{H} 63 \mathrm{~B}$ & 109.1 \\
\hline $\mathrm{C} 14-\mathrm{C} 19-\mathrm{H} 19$ & 119.7 & $\mathrm{H} 63 \mathrm{~A}-\mathrm{C} 63-\mathrm{H} 63 \mathrm{~B}$ & 107.8 \\
\hline $\mathrm{C} 22-\mathrm{C} 21-\mathrm{H} 21 \mathrm{~A}$ & 109.5 & C65-C64-C69 & $117.7(3)$ \\
\hline $\mathrm{C} 22-\mathrm{C} 21-\mathrm{H} 21 \mathrm{~B}$ & 109.5 & $\mathrm{C} 65-\mathrm{C} 64-\mathrm{C} 63$ & $121.0(3)$ \\
\hline $\mathrm{H} 21 \mathrm{~A}-\mathrm{C} 21-\mathrm{H} 21 \mathrm{~B}$ & 109.5 & $\mathrm{C} 69-\mathrm{C} 64-\mathrm{C} 63$ & $121.3(3)$ \\
\hline $\mathrm{C} 22-\mathrm{C} 21-\mathrm{H} 21 \mathrm{C}$ & 109.5 & $\mathrm{C} 64-\mathrm{C} 65-\mathrm{C} 66$ & $121.4(3)$ \\
\hline $\mathrm{H} 21 \mathrm{~A}-\mathrm{C} 21-\mathrm{H} 21 \mathrm{C}$ & 109.5 & $\mathrm{C} 64-\mathrm{C} 65-\mathrm{H} 65$ & 119.3 \\
\hline $\mathrm{H} 21 \mathrm{~B}-\mathrm{C} 21-\mathrm{H} 21 \mathrm{C}$ & 109.5 & С66-C65-H65 & 119.3 \\
\hline N3-C22-C21 & $108.4(3)$ & C67-C66-C65 & $120.7(4)$ \\
\hline $\mathrm{N} 3-\mathrm{C} 22-\mathrm{C} 23$ & $109.1(3)$ & C67-C66-H66 & 119.7 \\
\hline $\mathrm{C} 21-\mathrm{C} 22-\mathrm{C} 23$ & $113.6(3)$ & C65-C66-H66 & 119.7 \\
\hline $\mathrm{N} 3-\mathrm{C} 22-\mathrm{H} 22$ & 108.5 & C66-C67-C68 & $118.7(4)$ \\
\hline $\mathrm{C} 21-\mathrm{C} 22-\mathrm{H} 22$ & 108.5 & C66-C67-H67 & 120.6 \\
\hline $\mathrm{C} 23-\mathrm{C} 22-\mathrm{H} 22$ & 108.5 & C68-C67-H67 & 120.6 \\
\hline $\mathrm{C} 24-\mathrm{C} 23-\mathrm{C} 22$ & $112.2(3)$ & $\mathrm{C} 69-\mathrm{C} 68-\mathrm{C} 67$ & $120.7(3)$ \\
\hline $\mathrm{C} 24-\mathrm{C} 23-\mathrm{H} 23 \mathrm{~A}$ & 109.2 & $\mathrm{C} 69-\mathrm{C} 68-\mathrm{H} 68$ & 119.7 \\
\hline $\mathrm{C} 22-\mathrm{C} 23-\mathrm{H} 23 \mathrm{~A}$ & 109.2 & C67-C68-H68 & 119.7 \\
\hline $\mathrm{C} 24-\mathrm{C} 23-\mathrm{H} 23 \mathrm{~B}$ & 109.2 & C68-C69-C64 & $120.8(3)$ \\
\hline $\mathrm{C} 22-\mathrm{C} 23-\mathrm{H} 23 \mathrm{~B}$ & 109.2 & C68-C69-H69 & 119.6 \\
\hline
\end{tabular}




\begin{tabular}{|c|c|c|c|}
\hline $\mathrm{H} 23 \mathrm{~A}-\mathrm{C} 23-\mathrm{H} 23 \mathrm{~B}$ & 107.9 & C64-C69-H69 & 119.6 \\
\hline $\mathrm{C} 1-\mathrm{C} 2-\mathrm{C} 3-\mathrm{C} 4$ & $-57.2(4)$ & $\mathrm{N} 4-\mathrm{C} 32-\mathrm{C} 33-\mathrm{C} 34$ & $-179.0(3)$ \\
\hline $\mathrm{N} 1-\mathrm{C} 2-\mathrm{C} 3-\mathrm{C} 4$ & $-177.0(3)$ & $\mathrm{C} 31-\mathrm{C} 32-\mathrm{C} 33-\mathrm{C} 34$ & $-57.9(4)$ \\
\hline $\mathrm{C} 2-\mathrm{C} 3-\mathrm{C} 4-\mathrm{C} 5$ & $-69.3(4)$ & $\mathrm{C} 32-\mathrm{C} 33-\mathrm{C} 34-\mathrm{C} 35$ & $-66.7(4)$ \\
\hline $\mathrm{C} 2-\mathrm{C} 3-\mathrm{C} 4-\mathrm{C} 9$ & $106.9(4)$ & $\mathrm{C} 32-\mathrm{C} 33-\mathrm{C} 34-\mathrm{C} 39$ & $109.7(4)$ \\
\hline $\mathrm{C} 9-\mathrm{C} 4-\mathrm{C} 5-\mathrm{C} 6$ & $1.0(5)$ & $\mathrm{C} 39-\mathrm{C} 34-\mathrm{C} 35-\mathrm{C} 36$ & $0.1(5)$ \\
\hline $\mathrm{C} 3-\mathrm{C} 4-\mathrm{C} 5-\mathrm{C} 6$ & $177.3(3)$ & $\mathrm{C} 33-\mathrm{C} 34-\mathrm{C} 35-\mathrm{C} 36$ & $176.6(3)$ \\
\hline $\mathrm{C} 4-\mathrm{C} 5-\mathrm{C} 6-\mathrm{C} 7$ & $-0.4(5)$ & $\mathrm{C} 34-\mathrm{C} 35-\mathrm{C} 36-\mathrm{C} 37$ & $-0.8(6)$ \\
\hline $\mathrm{C} 5-\mathrm{C} 6-\mathrm{C} 7-\mathrm{C} 8$ & $0.1(6)$ & $\mathrm{C} 35-\mathrm{C} 36-\mathrm{C} 37-\mathrm{C} 38$ & $0.7(6)$ \\
\hline $\mathrm{C} 6-\mathrm{C} 7-\mathrm{C} 8-\mathrm{C} 9$ & $-0.5(6)$ & $\mathrm{C} 36-\mathrm{C} 37-\mathrm{C} 38-\mathrm{C} 39$ & $0.1(5)$ \\
\hline $\mathrm{C} 7-\mathrm{C} 8-\mathrm{C} 9-\mathrm{C} 4$ & $1.2(6)$ & $\mathrm{C} 35-\mathrm{C} 34-\mathrm{C} 39-\mathrm{C} 38$ & $0.7(5)$ \\
\hline $\mathrm{C} 5-\mathrm{C} 4-\mathrm{C} 9-\mathrm{C} 8$ & $-1.4(5)$ & $\mathrm{C} 33-\mathrm{C} 34-\mathrm{C} 39-\mathrm{C} 38$ & $-175.8(3)$ \\
\hline $\mathrm{C} 3-\mathrm{C} 4-\mathrm{C} 9-\mathrm{C} 8$ & $-177.7(3)$ & $\mathrm{C} 37-\mathrm{C} 38-\mathrm{C} 39-\mathrm{C} 34$ & $-0.8(5)$ \\
\hline $\mathrm{C} 11-\mathrm{C} 12-\mathrm{C} 13-\mathrm{C} 14$ & $-60.7(4)$ & N5-C52-C53-C54 & $179.3(3)$ \\
\hline $\mathrm{N} 2-\mathrm{C} 12-\mathrm{C} 13-\mathrm{C} 14$ & $176.7(3)$ & $\mathrm{C} 51-\mathrm{C} 52-\mathrm{C} 53-\mathrm{C} 54$ & $-60.8(4)$ \\
\hline $\mathrm{C} 12-\mathrm{C} 13-\mathrm{C} 14-\mathrm{C} 15$ & $115.4(4)$ & $\mathrm{C} 52-\mathrm{C} 53-\mathrm{C} 54-\mathrm{C} 59$ & $-52.7(4)$ \\
\hline $\mathrm{C} 12-\mathrm{C} 13-\mathrm{C} 14-\mathrm{C} 19$ & $-61.9(5)$ & $\mathrm{C} 52-\mathrm{C} 53-\mathrm{C} 54-\mathrm{C} 55$ & $127.5(3)$ \\
\hline $\mathrm{C} 19-\mathrm{C} 14-\mathrm{C} 15-\mathrm{C} 16$ & $-0.4(7)$ & C59-C54-C55-C56 & $-2.2(5)$ \\
\hline $\mathrm{C} 13-\mathrm{C} 14-\mathrm{C} 15-\mathrm{C} 16$ & $-177.8(5)$ & $\mathrm{C} 53-\mathrm{C} 54-\mathrm{C} 55-\mathrm{C} 56$ & $177.6(3)$ \\
\hline $\mathrm{C} 14-\mathrm{C} 15-\mathrm{C} 16-\mathrm{C} 17$ & $0.1(8)$ & $\mathrm{C} 54-\mathrm{C} 55-\mathrm{C} 56-\mathrm{C} 57$ & $0.8(6)$ \\
\hline $\mathrm{C} 15-\mathrm{C} 16-\mathrm{C} 17-\mathrm{C} 18$ & $1.6(8)$ & $\mathrm{C} 55-\mathrm{C} 56-\mathrm{C} 57-\mathrm{C} 58$ & $1.5(6)$ \\
\hline $\mathrm{C} 16-\mathrm{C} 17-\mathrm{C} 18-\mathrm{C} 19$ & $-3.0(7)$ & $\mathrm{C} 56-\mathrm{C} 57-\mathrm{C} 58-\mathrm{C} 59$ & $-2.4(5)$ \\
\hline $\mathrm{C} 17-\mathrm{C} 18-\mathrm{C} 19-\mathrm{C} 14$ & $2.8(7)$ & $\mathrm{C} 57-\mathrm{C} 58-\mathrm{C} 59-\mathrm{C} 54$ & $1.0(5)$ \\
\hline $\mathrm{C} 15-\mathrm{C} 14-\mathrm{C} 19-\mathrm{C} 18$ & $-1.1(6)$ & $\mathrm{C} 55-\mathrm{C} 54-\mathrm{C} 59-\mathrm{C} 58$ & $1.3(5)$ \\
\hline $\mathrm{C} 13-\mathrm{C} 14-\mathrm{C} 19-\mathrm{C} 18$ & $176.3(4)$ & $\mathrm{C} 53-\mathrm{C} 54-\mathrm{C} 59-\mathrm{C} 58$ & $-178.5(3)$ \\
\hline $\mathrm{N} 3-\mathrm{C} 22-\mathrm{C} 23-\mathrm{C} 24$ & $177.6(3)$ & N6-C62-C63-C64 & $178.6(3)$ \\
\hline $\mathrm{C} 21-\mathrm{C} 22-\mathrm{C} 23-\mathrm{C} 24$ & $-61.3(4)$ & $\mathrm{C} 61-\mathrm{C} 62-\mathrm{C} 63-\mathrm{C} 64$ & $-60.7(4)$ \\
\hline $\mathrm{C} 22-\mathrm{C} 23-\mathrm{C} 24-\mathrm{C} 29$ & $113.2(4)$ & $\mathrm{C} 62-\mathrm{C} 63-\mathrm{C} 64-\mathrm{C} 65$ & $-63.9(4)$ \\
\hline $\mathrm{C} 22-\mathrm{C} 23-\mathrm{C} 24-\mathrm{C} 25$ & $-65.1(4)$ & $\mathrm{C} 62-\mathrm{C} 63-\mathrm{C} 64-\mathrm{C} 69$ & $114.7(4)$ \\
\hline $\mathrm{C} 29-\mathrm{C} 24-\mathrm{C} 25-\mathrm{C} 26$ & $0.2(5)$ & $\mathrm{C} 69-\mathrm{C} 64-\mathrm{C} 65-\mathrm{C} 66$ & $0.3(5)$ \\
\hline $\mathrm{C} 23-\mathrm{C} 24-\mathrm{C} 25-\mathrm{C} 26$ & $178.6(3)$ & $\mathrm{C} 63-\mathrm{C} 64-\mathrm{C} 65-\mathrm{C} 66$ & $179.0(3)$ \\
\hline $\mathrm{C} 24-\mathrm{C} 25-\mathrm{C} 26-\mathrm{C} 27$ & $-0.7(6)$ & $\mathrm{C} 64-\mathrm{C} 65-\mathrm{C} 66-\mathrm{C} 67$ & $-0.6(6)$ \\
\hline $\mathrm{C} 25-\mathrm{C} 26-\mathrm{C} 27-\mathrm{C} 28$ & $0.3(6)$ & $\mathrm{C} 65-\mathrm{C} 66-\mathrm{C} 67-\mathrm{C} 68$ & $0.1(6)$ \\
\hline $\mathrm{C} 26-\mathrm{C} 27-\mathrm{C} 28-\mathrm{C} 29$ & $0.6(5)$ & C66-C67-C68-C69 & $0.8(6)$ \\
\hline $\mathrm{C} 25-\mathrm{C} 24-\mathrm{C} 29-\mathrm{C} 28$ & $0.7(5)$ & $\mathrm{C} 67-\mathrm{C} 68-\mathrm{C} 69-\mathrm{C} 64$ & $-1.1(6)$ \\
\hline $\mathrm{C} 23-\mathrm{C} 24-\mathrm{C} 29-\mathrm{C} 28$ & $-177.7(3)$ & $\mathrm{C} 65-\mathrm{C} 64-\mathrm{C} 69-\mathrm{C} 68$ & $0.5(5)$ \\
\hline $\mathrm{C} 27-\mathrm{C} 28-\mathrm{C} 29-\mathrm{C} 24$ & $-1.1(6)$ & $\mathrm{C} 63-\mathrm{C} 64-\mathrm{C} 69-\mathrm{C} 68$ & $-178.1(3)$ \\
\hline
\end{tabular}

Hydrogen-bond geometry $\left(A,{ }^{\circ}\right)$ for (I)

\begin{tabular}{lllll}
\hline$D-\mathrm{H} \cdots A$ & $D-\mathrm{H}$ & $\mathrm{H} \cdots A$ & $D \cdots A$ & $D-\mathrm{H} \cdots A$ \\
\hline $\mathrm{N} 1-\mathrm{H} 1 A \cdots \mathrm{Cl} 5^{\mathrm{i}}$ & 0.91 & 2.40 & $3.304(3)$ & 171 \\
$\mathrm{~N} 1-\mathrm{H} 1 B \cdots \mathrm{Cl} 5^{\mathrm{ii}}$ & 0.91 & 2.27 & $3.177(3)$ & 171 \\
$\mathrm{~N} 1-\mathrm{H} 1 C \cdots \mathrm{Cl} 6^{\mathrm{i}}$ & 0.91 & 2.28 & $3.181(3)$ & 169 \\
$\mathrm{~N} 2-\mathrm{H} 2 A \cdots \mathrm{Cl} 6^{\mathrm{iii}}$ & 0.91 & 2.27 & $3.179(3)$ & 174 \\
$\mathrm{~N} 2-\mathrm{H} 2 B \cdots \mathrm{Cl} 1^{\mathrm{iv}}$ & 0.91 & 2.43 & $3.215(3)$ & 145 \\
$\mathrm{~N} 2-\mathrm{H} 2 C \cdots \mathrm{Cl} 6^{\mathrm{i}}$ & 0.91 & 2.37 & $3.233(3)$ & 158 \\
$\mathrm{~N} 3-\mathrm{H} 3 A \cdots \mathrm{Cl} 1^{\mathrm{iv}}$ & 0.91 & 2.28 & $3.151(3)$ & 159 \\
$\mathrm{~N} 3-\mathrm{H} 3 B \cdots \mathrm{Cl} 1$ & 0.91 & 2.22 & $3.118(3)$ & 168 \\
$\mathrm{~N} 3-\mathrm{H} 3 C \cdots \mathrm{Cl} 2$ & 0.91 & 2.27 & $3.171(3)$ & 169 \\
$\mathrm{~N} 4-\mathrm{H} 4 A \cdots \mathrm{Cl} 2$ & 0.91 & 2.25 & $3.149(3)$ & 168 \\
$\mathrm{~N} 4-\mathrm{H} 4 B \cdots \mathrm{Cl} 3$ & 0.91 & 2.26 & $3.164(3)$ & 172 \\
$\mathrm{~N} 4-\mathrm{H} 4 C \cdots \mathrm{Cl} 2^{\mathrm{v}}$ & 0.91 & 2.37 & $3.271(3)$ &
\end{tabular}




\begin{tabular}{lllll}
$\mathrm{N} 5-\mathrm{H} 5 A \cdots \mathrm{Cl} 3$ & 0.91 & 2.27 & $3.159(3)$ & 165 \\
$\mathrm{~N} 5-\mathrm{H} 5 B \cdots \mathrm{Cl} 4$ & 0.91 & 2.36 & $3.196(3)$ & 153 \\
$\mathrm{~N} 5-\mathrm{H} 5 C \cdots \mathrm{Cl}{ }^{\text {iv }}$ & 0.91 & 2.33 & $3.233(3)$ & 170 \\
$\mathrm{~N} 6-\mathrm{H} 6 A \cdots \mathrm{Cl} 4$ & 0.91 & 2.22 & $3.117(3)$ & 167 \\
$\mathrm{~N} 6-\mathrm{H} 6 B \cdots \mathrm{Cl} 5^{\mathrm{v}}$ & 0.91 & 2.27 & $3.168(3)$ & 169 \\
$\mathrm{~N} 6-\mathrm{H} 6 C \cdots \mathrm{Cl} 4^{\mathrm{v}}$ & 0.91 & 2.29 & $3.158(3)$ & 161 \\
\hline
\end{tabular}

Symmetry codes: (i) $x, y, z+1$; (ii) $x-1, y, z+1$; (iii) $x+1, y, z+1$; (iv) $x+1, y, z$; (v) $x-1, y, z$.

(II)

\section{Crystal data}

$$
\begin{aligned}
& \mathrm{C}_{9} \mathrm{H}_{14} \mathrm{~N} \cdot \mathrm{Br} \\
& M_{r}=216.12 \\
& \text { Monoclinic, } P 2_{1} \\
& \text { Hall symbol: } \mathrm{P} 2 \mathrm{yb} \\
& a=5.2366(4) \AA \\
& b=8.4264(5) \AA \\
& c=11.3895(8) \AA \\
& \beta=94.079(2)^{\circ} \\
& V=501.30(6) \AA^{3} \\
& Z=2
\end{aligned}
$$

\section{Data collection}

Rigaku Saturn724+ (2x2 bin mode) diffractometer

Radiation source: fine-focus sealed tube Graphite monochromator

Detector resolution: 28.5714 pixels $\mathrm{mm}^{-1}$

profile data from $\omega$-scans

Absorption correction: multi-scan

CrystalClear-SM Expert 3.1 (Rigaku, 2013)

$T_{\min }=0.711, T_{\max }=1.000$

\section{Refinement}

Refinement on $F^{2}$

Least-squares matrix: full

$R\left[F^{2}>2 \sigma\left(F^{2}\right)\right]=0.020$

$w R\left(F^{2}\right)=0.052$

$S=1.08$

2383 reflections

115 parameters

1 restraint

Primary atom site location: structure-invariant direct methods

Secondary atom site location: difference Fourier map Hydrogen site location: inferred from neighbouring sites
$F(000)=220$

$D_{\mathrm{x}}=1.432 \mathrm{Mg} \mathrm{m}^{-3}$

Mo $K \alpha$ radiation, $\lambda=0.71069 \AA$

Cell parameters from 5999 reflections

$\theta=3.0-27.5^{\circ}$

$\mu=4.04 \mathrm{~mm}^{-1}$

$T=100 \mathrm{~K}$

Blade, colourless

$0.25 \times 0.18 \times 0.12 \mathrm{~mm}$

5432 measured reflections

2383 independent reflections

2343 reflections with $I>2 \sigma(I)$

$R_{\text {int }}=0.019$

$\theta_{\max }=30.1^{\circ}, \theta_{\min }=3.0^{\circ}$

$h=-7 \rightarrow 7$

$k=-11 \rightarrow 10$

$l=-16 \rightarrow 15$

$\mathrm{H}$ atoms treated by a mixture of independent and constrained refinement

$w=1 /\left[\sigma^{2}\left(F_{\mathrm{o}}^{2}\right)+(0.0329 P)^{2}+0.1173 P\right]$

where $P=\left(F_{\mathrm{o}}{ }^{2}+2 F_{\mathrm{c}}{ }^{2}\right) / 3$

$(\Delta / \sigma)_{\max }=0.001$

$\Delta \rho_{\max }=0.29 \mathrm{e} \AA^{-3}$

$\Delta \rho_{\min }=-0.39$ e $\AA^{-3}$

Extinction correction: SHELXL,

$\mathrm{Fc}^{*}=\mathrm{kFc}\left[1+0.001 \times \mathrm{Fc}^{2} \lambda^{3} / \sin (2 \theta)\right]^{-1 / 4}$

Extinction coefficient: 0.045 (3)

Absolute structure: Flack H D (1983), Acta Cryst.

A39, 876-881

Absolute structure parameter: 0.003 (10)

\section{Special details}

Refinement

Refinement of $F^{2}$ against ALL reflections. The weighted $R$-factor $w R$ and goodness of fit $S$ are based on $F^{2}$, conventional $R$-factors $R$ are based on $F$, with $F$ set to zero for negative $F^{2}$. The threshold expression of $F^{2}>2 \sigma\left(F^{2}\right)$ is used only for calculating $R$-factors(gt) etc. and is not relevant to the choice of reflections for refinement. $R$-factors based on $F^{2}$ are statistically about twice as large as those based on $F$, and $R$ - factors based on ALL data will be even larger. 
Fractional atomic coordinates and isotropic or equivalent isotropic displacement parameters $\left(\AA^{2}\right)$ for (II)

\begin{tabular}{|c|c|c|c|c|}
\hline & $x$ & $y$ & $z$ & $U_{\text {iso }} * / U_{\text {eq }}$ \\
\hline $\mathrm{Br} 1$ & $0.08467(3)$ & $0.71309(4)$ & $0.418045(13)$ & $0.02270(7)$ \\
\hline N1 & $0.6129(4)$ & $0.5569(2)$ & $0.55110(15)$ & $0.0206(3)$ \\
\hline $\mathrm{C} 1$ & $0.6190(5)$ & $0.7824(3)$ & $0.6887(2)$ & $0.0267(5)$ \\
\hline $\mathrm{H} 1 \mathrm{~A}$ & 0.7315 & 0.8410 & 0.6389 & $0.040^{*}$ \\
\hline H1B & 0.6541 & 0.8148 & 0.7709 & $0.040^{*}$ \\
\hline $\mathrm{H} 1 \mathrm{C}$ & 0.4400 & 0.8055 & 0.6636 & $0.040^{*}$ \\
\hline $\mathrm{C} 2$ & $0.6679(4)$ & $0.6056(3)$ & 0.67731 (19) & $0.0198(4)$ \\
\hline $\mathrm{H} 2$ & 0.8533 & 0.5854 & 0.6994 & $0.024 *$ \\
\hline $\mathrm{C} 3$ & $0.5097(3)$ & $0.5028(2)$ & $0.75640(17)$ & 0.0202 (4) \\
\hline $\mathrm{H} 3 \mathrm{~A}$ & 0.3326 & 0.5442 & 0.7546 & $0.024 *$ \\
\hline H3B & 0.5024 & 0.3927 & 0.7260 & $0.024 *$ \\
\hline $\mathrm{C} 4$ & $0.6258(3)$ & $0.5028(2)$ & 0.88157 (17) & $0.0187(3)$ \\
\hline $\mathrm{C} 5$ & 0.8463 (4) & $0.4144(2)$ & 0.91057 (17) & 0.0209 (4) \\
\hline H5 & 0.9208 & 0.3539 & 0.8514 & $0.025^{*}$ \\
\hline C6 & 0.9585 (4) & $0.4134(3)$ & $1.02495(18)$ & $0.0237(4)$ \\
\hline H6 & 1.1085 & 0.3524 & 1.0435 & $0.028^{*}$ \\
\hline $\mathrm{C} 7$ & $0.8511(4)$ & $0.5017(3)$ & 1.11225 (19) & $0.0262(4)$ \\
\hline H7 & 0.9281 & 0.5019 & 1.1903 & $0.031 *$ \\
\hline $\mathrm{C} 8$ & $0.6311(4)$ & $0.5894(3)$ & 1.08438 (19) & $0.0269(4)$ \\
\hline H8 & 0.5567 & 0.6492 & 1.1439 & $0.032 *$ \\
\hline C9 & 0.5187 (4) & $0.5905(3)$ & $0.97025(18)$ & $0.0240(4)$ \\
\hline H9 & 0.3680 & 0.6511 & 0.9522 & $0.029 *$ \\
\hline $\mathrm{H} 1 \mathrm{~N}$ & $0.649(5)$ & $0.461(4)$ & $0.542(2)$ & $0.030(7)^{*}$ \\
\hline $\mathrm{H} 2 \mathrm{~N}$ & $0.724(5)$ & $0.604(4)$ & $0.501(3)$ & $0.033(7)^{*}$ \\
\hline $\mathrm{H} 3 \mathrm{~N}$ & $0.442(6)$ & $0.576(4)$ & $0.524(3)$ & $0.038(8)^{*}$ \\
\hline
\end{tabular}

Atomic displacement parameters $\left(\AA^{2}\right)$ for (II)

\begin{tabular}{lllllll}
\hline & $U^{11}$ & $U^{22}$ & $U^{33}$ & $U^{12}$ & $U^{13}$ & $U^{23}$ \\
\hline Br1 & $0.02399(10)$ & $0.02052(10)$ & $0.02300(10)$ & $-0.00361(9)$ & $-0.00246(6)$ & $0.00244(9)$ \\
N1 & $0.0249(8)$ & $0.0182(8)$ & $0.0183(7)$ & $0.0019(6)$ & $-0.0017(6)$ & $0.0003(6)$ \\
C1 & $0.0379(14)$ & $0.0202(11)$ & $0.0209(10)$ & $-0.0013(9)$ & $-0.0059(9)$ & $-0.0007(8)$ \\
C2 & $0.0210(9)$ & $0.0203(10)$ & $0.0174(9)$ & $-0.0024(8)$ & $-0.0029(7)$ & $0.0023(8)$ \\
C3 & $0.0175(8)$ & $0.0216(9)$ & $0.0210(8)$ & $-0.0022(7)$ & $-0.0029(6)$ & $0.0018(7)$ \\
C4 & $0.0185(8)$ & $0.0185(9)$ & $0.0191(8)$ & $-0.0040(7)$ & $0.0015(6)$ & $0.0013(7)$ \\
C5 & $0.0217(9)$ & $0.0221(9)$ & $0.0190(8)$ & $0.0006(7)$ & $0.0018(7)$ & $0.0017(7)$ \\
C6 & $0.0218(9)$ & $0.0271(10)$ & $0.0218(9)$ & $-0.0007(7)$ & $-0.0021(7)$ & $0.0038(7)$ \\
C7 & $0.0318(10)$ & $0.0258(10)$ & $0.0204(8)$ & $-0.0069(8)$ & $-0.0030(8)$ & $0.0029(8)$ \\
C8 & $0.0374(11)$ & $0.0222(10)$ & $0.0217(9)$ & $-0.0001(8)$ & $0.0055(8)$ & $-0.0024(8)$ \\
C9 & $0.0257(9)$ & $0.0206(9)$ & $0.0258(10)$ & $0.0035(7)$ & $0.0029(8)$ & $0.0015(8)$ \\
\hline
\end{tabular}

Geometric parameters $\left(\AA,{ }^{\circ}\right)$ for (II)

\begin{tabular}{llll}
\hline $\mathrm{N} 1-\mathrm{C} 2$ & $1.503(3)$ & $\mathrm{C} 3-\mathrm{H} 3 \mathrm{~B}$ & 0.9900 \\
$\mathrm{~N} 1-\mathrm{H} 1 \mathrm{~N}$ & $0.83(3)$ & $\mathrm{C} 4-\mathrm{C} 5$ & $1.394(3)$ \\
$\mathrm{N} 1-\mathrm{H} 2 \mathrm{~N}$ & $0.93(3)$ & $\mathrm{C} 4-\mathrm{C} 9$ & $1.400(3)$ \\
$\mathrm{N} 1-\mathrm{H} 3 \mathrm{~N}$ & $0.94(3)$ & $\mathrm{C} 5-\mathrm{C} 6$ & $1.391(3)$ \\
$\mathrm{C} 1-\mathrm{C} 2$ & $1.519(3)$ & $\mathrm{C} 5-\mathrm{H} 5$ & 0.9500 \\
$\mathrm{C} 1-\mathrm{H} 1 \mathrm{~A}$ & 0.9800 & $\mathrm{C} 6-\mathrm{C} 7$ & $1.392(3)$ \\
$\mathrm{C} 1-\mathrm{H} 1 \mathrm{~B}$ & 0.9800 & $\mathrm{C} 6-\mathrm{H} 6$ & 0.9500 \\
$\mathrm{C} 1-\mathrm{H} 1 \mathrm{C}$ & 0.9800 & $\mathrm{C} 7-\mathrm{C} 8$ & $1.386(3)$
\end{tabular}


supplementary materials

\begin{tabular}{|c|c|c|c|}
\hline $\mathrm{C} 2-\mathrm{C} 3$ & $1.534(3)$ & $\mathrm{C} 7-\mathrm{H} 7$ & 0.9500 \\
\hline $\mathrm{C} 2-\mathrm{H} 2$ & 1.0000 & $\mathrm{C} 8-\mathrm{C} 9$ & $1.388(3)$ \\
\hline $\mathrm{C} 3-\mathrm{C} 4$ & $1.509(3)$ & $\mathrm{C} 8-\mathrm{H} 8$ & 0.9500 \\
\hline $\mathrm{C} 3-\mathrm{H} 3 \mathrm{~A}$ & 0.9900 & C9-H9 & 0.9500 \\
\hline $\mathrm{C} 2-\mathrm{N} 1-\mathrm{H} 1 \mathrm{~N}$ & $110.3(19)$ & $\mathrm{C} 4-\mathrm{C} 3-\mathrm{H} 3 \mathrm{~B}$ & 109.5 \\
\hline $\mathrm{C} 2-\mathrm{N} 1-\mathrm{H} 2 \mathrm{~N}$ & $112.5(18)$ & $\mathrm{C} 2-\mathrm{C} 3-\mathrm{H} 3 \mathrm{~B}$ & 109.5 \\
\hline $\mathrm{H} 1 \mathrm{~N}-\mathrm{N} 1-\mathrm{H} 2 \mathrm{~N}$ & $100(2)$ & $\mathrm{H} 3 \mathrm{~A}-\mathrm{C} 3-\mathrm{H} 3 \mathrm{~B}$ & 108.1 \\
\hline $\mathrm{C} 2-\mathrm{N} 1-\mathrm{H} 3 \mathrm{~N}$ & $112.6(19)$ & $\mathrm{C} 5-\mathrm{C} 4-\mathrm{C} 9$ & $118.60(18)$ \\
\hline $\mathrm{H} 1 \mathrm{~N}-\mathrm{N} 1-\mathrm{H} 3 \mathrm{~N}$ & $110(3)$ & $\mathrm{C} 5-\mathrm{C} 4-\mathrm{C} 3$ & $119.65(18)$ \\
\hline $\mathrm{H} 2 \mathrm{~N}-\mathrm{N} 1-\mathrm{H} 3 \mathrm{~N}$ & $111(3)$ & $\mathrm{C} 9-\mathrm{C} 4-\mathrm{C} 3$ & $121.76(18)$ \\
\hline $\mathrm{C} 2-\mathrm{C} 1-\mathrm{H} 1 \mathrm{~A}$ & 109.5 & $\mathrm{C} 6-\mathrm{C} 5-\mathrm{C} 4$ & $120.84(18)$ \\
\hline $\mathrm{C} 2-\mathrm{C} 1-\mathrm{H} 1 \mathrm{~B}$ & 109.5 & $\mathrm{C} 6-\mathrm{C} 5-\mathrm{H} 5$ & 119.6 \\
\hline $\mathrm{H} 1 \mathrm{~A}-\mathrm{C} 1-\mathrm{H} 1 \mathrm{~B}$ & 109.5 & $\mathrm{C} 4-\mathrm{C} 5-\mathrm{H} 5$ & 119.6 \\
\hline $\mathrm{C} 2-\mathrm{C} 1-\mathrm{H} 1 \mathrm{C}$ & 109.5 & $\mathrm{C} 5-\mathrm{C} 6-\mathrm{C} 7$ & $120.05(19)$ \\
\hline $\mathrm{H} 1 \mathrm{~A}-\mathrm{C} 1-\mathrm{H} 1 \mathrm{C}$ & 109.5 & $\mathrm{C} 5-\mathrm{C} 6-\mathrm{H} 6$ & 120.0 \\
\hline $\mathrm{H} 1 \mathrm{~B}-\mathrm{C} 1-\mathrm{H} 1 \mathrm{C}$ & 109.5 & $\mathrm{C} 7-\mathrm{C} 6-\mathrm{H} 6$ & 120.0 \\
\hline $\mathrm{N} 1-\mathrm{C} 2-\mathrm{C} 1$ & $109.1(2)$ & $\mathrm{C} 8-\mathrm{C} 7-\mathrm{C} 6$ & $119.52(19)$ \\
\hline $\mathrm{N} 1-\mathrm{C} 2-\mathrm{C} 3$ & $109.44(17)$ & $\mathrm{C} 8-\mathrm{C} 7-\mathrm{H} 7$ & 120.2 \\
\hline $\mathrm{C} 1-\mathrm{C} 2-\mathrm{C} 3$ & $113.74(19)$ & $\mathrm{C} 6-\mathrm{C} 7-\mathrm{H} 7$ & 120.2 \\
\hline $\mathrm{N} 1-\mathrm{C} 2-\mathrm{H} 2$ & 108.1 & $\mathrm{C} 7-\mathrm{C} 8-\mathrm{C} 9$ & $120.5(2)$ \\
\hline $\mathrm{C} 1-\mathrm{C} 2-\mathrm{H} 2$ & 108.1 & $\mathrm{C} 7-\mathrm{C} 8-\mathrm{H} 8$ & 119.7 \\
\hline $\mathrm{C} 3-\mathrm{C} 2-\mathrm{H} 2$ & 108.1 & $\mathrm{C} 9-\mathrm{C} 8-\mathrm{H} 8$ & 119.7 \\
\hline $\mathrm{C} 4-\mathrm{C} 3-\mathrm{C} 2$ & $110.88(16)$ & $\mathrm{C} 8-\mathrm{C} 9-\mathrm{C} 4$ & $120.46(19)$ \\
\hline $\mathrm{C} 4-\mathrm{C} 3-\mathrm{H} 3 \mathrm{~A}$ & 109.5 & $\mathrm{C} 8-\mathrm{C} 9-\mathrm{H} 9$ & 119.8 \\
\hline $\mathrm{C} 2-\mathrm{C} 3-\mathrm{H} 3 \mathrm{~A}$ & 109.5 & $\mathrm{C} 4-\mathrm{C} 9-\mathrm{H} 9$ & 119.8 \\
\hline $\mathrm{N} 1-\mathrm{C} 2-\mathrm{C} 3-\mathrm{C} 4$ & $158.84(17)$ & $\mathrm{C} 4-\mathrm{C} 5-\mathrm{C} 6-\mathrm{C} 7$ & $-0.1(3)$ \\
\hline $\mathrm{C} 1-\mathrm{C} 2-\mathrm{C} 3-\mathrm{C} 4$ & $-78.9(2)$ & $\mathrm{C} 5-\mathrm{C} 6-\mathrm{C} 7-\mathrm{C} 8$ & $0.5(3)$ \\
\hline $\mathrm{C} 2-\mathrm{C} 3-\mathrm{C} 4-\mathrm{C} 5$ & $-75.2(2)$ & $\mathrm{C} 6-\mathrm{C} 7-\mathrm{C} 8-\mathrm{C} 9$ & $-0.4(3)$ \\
\hline $\mathrm{C} 2-\mathrm{C} 3-\mathrm{C} 4-\mathrm{C} 9$ & $104.5(2)$ & $\mathrm{C} 7-\mathrm{C} 8-\mathrm{C} 9-\mathrm{C} 4$ & $0.1(3)$ \\
\hline $\mathrm{C} 9-\mathrm{C} 4-\mathrm{C} 5-\mathrm{C} 6$ & $-0.3(3)$ & $\mathrm{C} 5-\mathrm{C} 4-\mathrm{C} 9-\mathrm{C} 8$ & $0.3(3)$ \\
\hline $\mathrm{C} 3-\mathrm{C} 4-\mathrm{C} 5-\mathrm{C} 6$ & $179.42(19)$ & $\mathrm{C} 3-\mathrm{C} 4-\mathrm{C} 9-\mathrm{C} 8$ & $-179.38(18$ \\
\hline
\end{tabular}

Hydrogen-bond geometry ( $\left.A,{ }^{\circ}\right)$ for (II)

\begin{tabular}{lllll}
\hline$D-\mathrm{H} \cdots A$ & $D-\mathrm{H}$ & $\mathrm{H} \cdots A$ & $D \cdots A$ & $D-\mathrm{H} \cdots A$ \\
\hline $\mathrm{N} 1-\mathrm{H} 1 N \cdots \mathrm{Br} 1^{\mathrm{i}}$ & $0.83(3)$ & $2.54(3)$ & $3.3080(19)$ & $154(2)$ \\
$\mathrm{N} 1-\mathrm{H} 2 N \cdots \mathrm{Br} 1^{\mathrm{ii}}$ & $0.93(3)$ & $2.36(3)$ & $3.2656(19)$ & $165(3)$ \\
$\mathrm{N} 1-\mathrm{H} 3 N \cdots \mathrm{Br} 1$ & $0.94(3)$ & $2.44(3)$ & $3.3296(18)$ & $157(3)$ \\
\hline
\end{tabular}

Symmetry codes: (i) $-x+1, y-1 / 2,-z+1$; (ii) $x+1, y, z$. 